\title{
ANNOTATED CITES APPENDICES AND RESERVATIONS
}

PART 1 The CITES Iistings in Appendices I, II and III

PART 2 a record of the speciflc Reservations entered by Partios to CITES

$$
\text { by }
$$

Wildlife Trade Honitoring Unit

IUCN Conservation Monitoring Centre

Copyright (C) 1986. IUCN Conservation Monitoring Centre.

219c Huntingdon Road, Cambridge CB3 ODL, UK. 


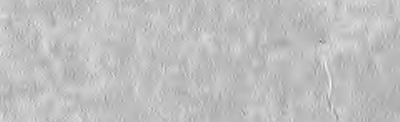

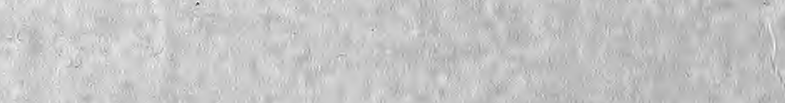

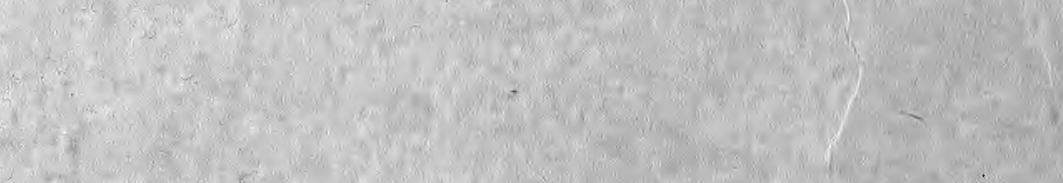

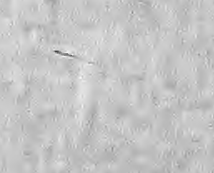

1are

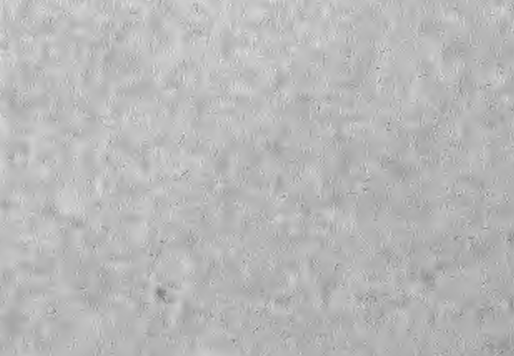

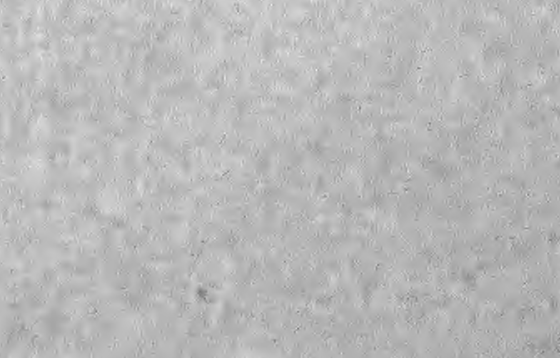

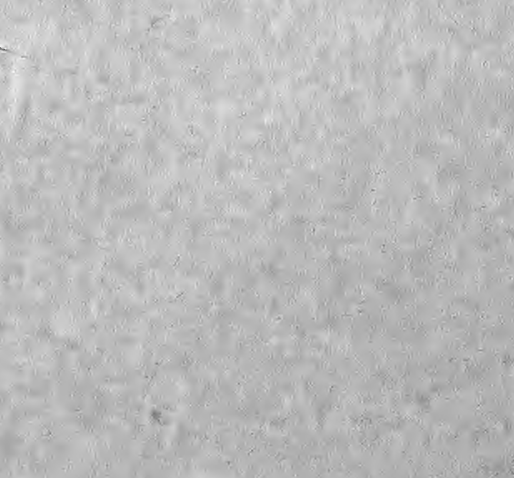

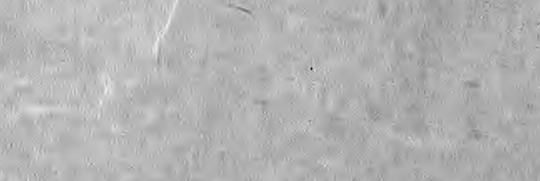

$5=$

(1)

mances

品

$(x+3)$

a.

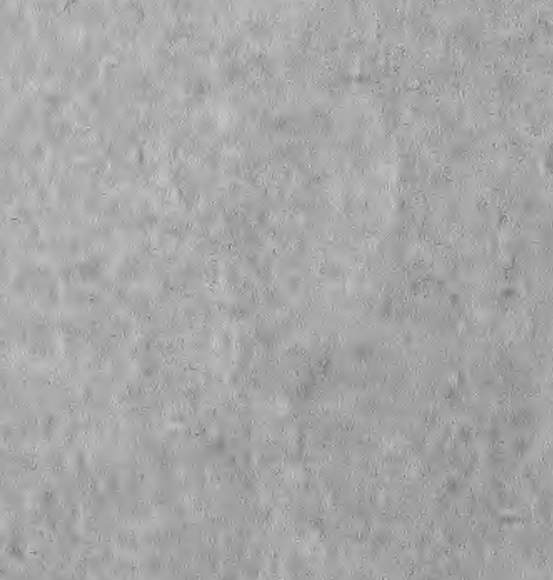

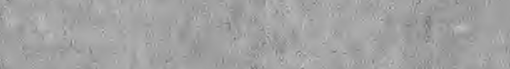

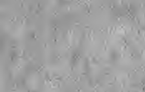

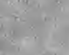

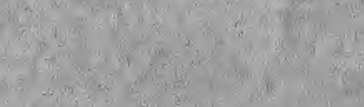

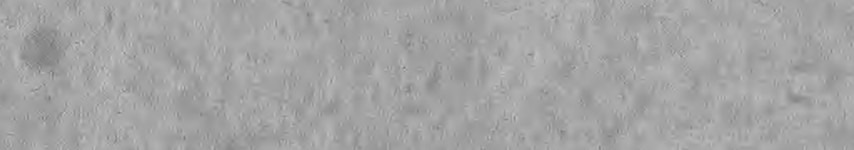

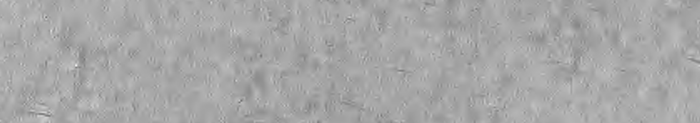

(ix)

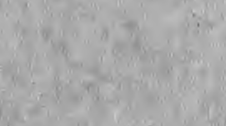

$\lim _{i=8} \lim ^{2}$

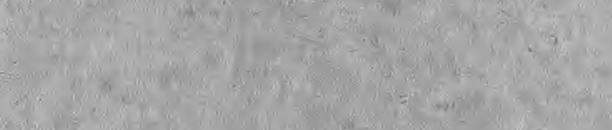

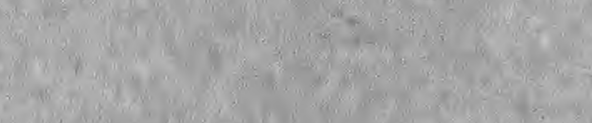
$5,2 x^{2}$

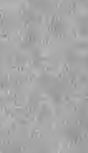
(2) $\rightarrow$ $\operatorname{lin}_{10}$ $\operatorname{lin}^{2}$

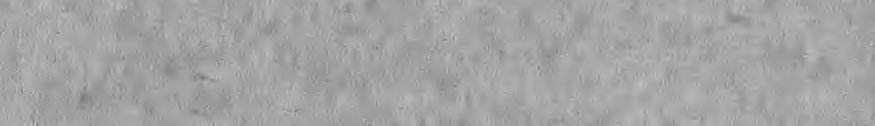

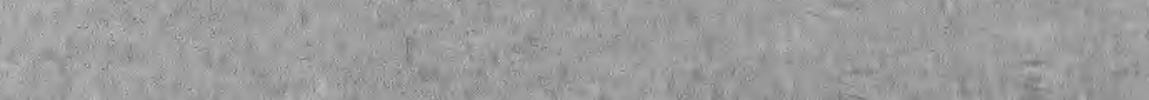

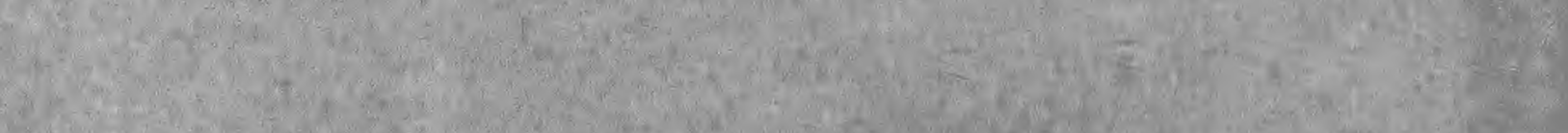
T. W.

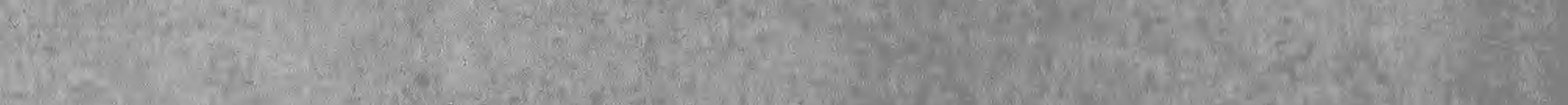

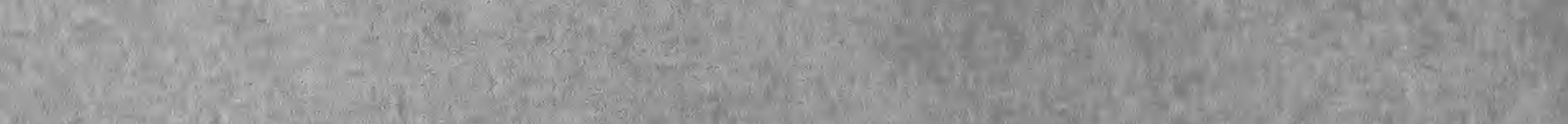
W. Whe

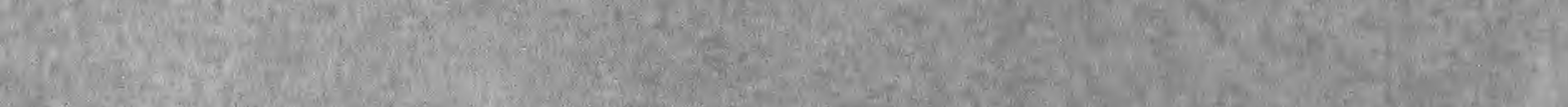
(6)

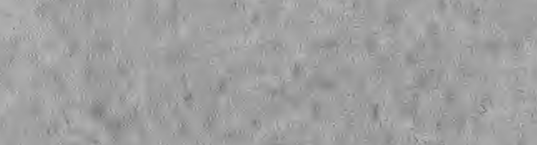

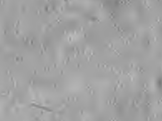

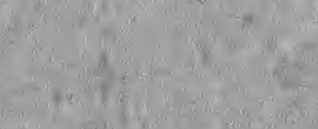

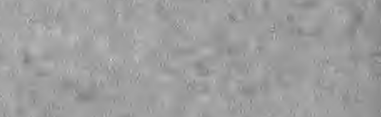

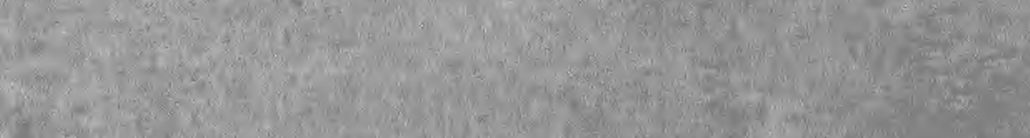
cond 
THE CITES LISTINGS IN APPENDICES I, II AND III

incorporating omendments made since

the Convention came into force

and English common names

The scientific names in these $l$ ists are based on the official Appendices $I$ and II as at 29 August 1986 and Appendix III as at 13 November 1986 , and on previous editions of the official Appendices, produced by the CITES Secretariat. The English common names are derived from many different sources but the main criterion for selection has been aptness. The majority are included in Common Names of Species Listed on Appendices I and II also produced by the CITES Secretariat.

Wildlife Trade Monitoring Unit

Copyright (C) 1986. IUCN Conservation Monitoring Centre. $219 \mathrm{c}$ Huntingdon Rosd, Cembridge CB3 ODL, UK. 


\section{Digitized by the Internet Archive in 2010 with funding from UNEP-WCMC, Cambridge}


Appendices I II - as at 29 August 1986

and Incorporating amendments wade since

the Convention came into ef foct

\section{INTERPRETATION}

1. Specles Included in these appendlces are raforred to:

a) by the nase of the pecles; or

b) as belng all of the specles included in algher taxon or destgnated part thereof.

2. The abbreviation "spp." is uaed to denote all spocles of a higher taxon.

3. Other references to taxe higher then secles are for the purposes of information or elasalfication only.

4. The abreviation "p.e." Is used to denote specles which are possbly oxtinct.

5. An asteriok (m) placed against the name of epecies or higher taxon Indicates that one or wore seographicalig eparate populations, subspecies or apecles of that pecles or taxon are Included in appendix I and that these populations, aubsecles or apecies are excluded from Appendix II.

6. Tro aterisk (*a) placed againet the name of apecles or higher taxon ladicates that one or wore geographlcally ceparte populations, subsecles or species of thet apecies or texon ere Included in Appendix II and thet these populations, subsecles or speclea ere excluded from Appendix I.

7. The aybol Q indicates that further detalls concerning that texon way bo found in the wotes os pages 30-33.

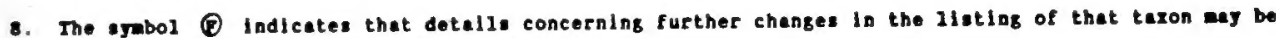
found in the Speclel Flore wotes on peges 33-34.

Q 1- Upgrediag of teron to Appendiz 1 .

- - - - 1 - Uptrading of part of taxon (1... populations, subapecies or epecien) to Append1 I I.

$1+2$ - Dowgrading of texon to Appendix II.

1 + Q. - Domgreding of part of taxon (1... populations, mubspectes or pecles) to uppecelix II.

(2) $Y 1$ = Incorporation of a population. aubapecies or spocles in algber taxon.

9

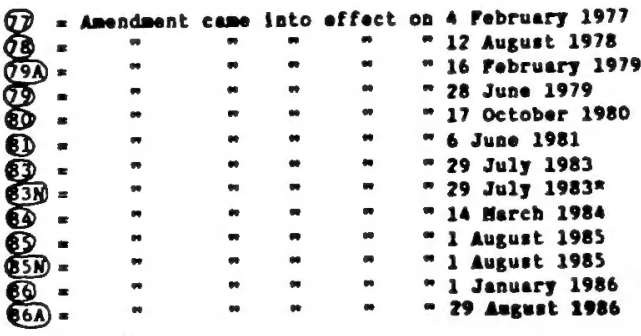

womenclatural ebages resultiag from Con. A.16 
Mame11.

\section{DOMOTREATA \\ Taebjeloseldae}

(72)

Zaglossus epp. Long-boaked ochldnes

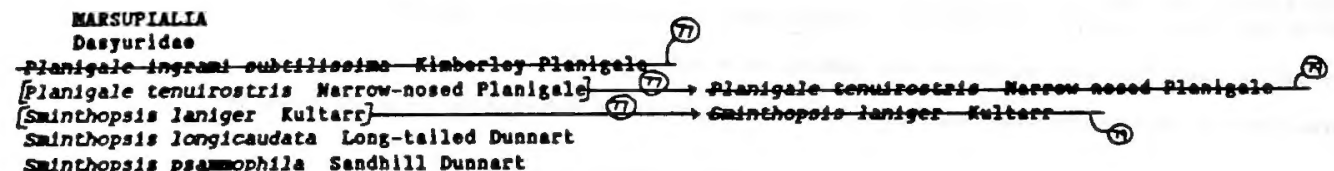

Triseclase

(3)

mylacinus cynocephalus pit. Toylecloe

Hracoblida

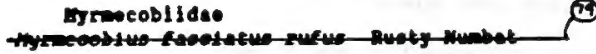

Perenidiae

cheropus caudatu p.o Plg-footed sendicoot

Peraneles bouganvilie Western Berred Bandleot

Prjlecenjlate

macrots lagotis Grater Bliby

macrotis levcurs Lassor silby

merangoridas

Phalanger aculatus Conon Spotted Cuscus

[ryulda squancaudace scely-talled posaug] $\rightarrow 7$ Phalanger orientalls Grey Cuscus (7)

[ourrarys parrus Lountein Pygey-Posub]

Toubetldee

Lasorhinus [g112esplei] kreffil Morthern Halry-

recopodidae

(7) nosed Uombet

7. Bettangla sp. Bettongs

Bettangla lesteurl Burrowing Bettong

Betcong1a penic111eta Brush-talled Bottons]

bettongla eroplca Northorn Bettong

Caloprynus crpestrs p.๑. Desert rat-kangaroo

T.

Dendrolagus bernetelanus Benatt's Ireo-Kangeroo bendrolagus inustus Grizzled Tree-Kangaroo Dendrolagus lumolted Cenholtz' Tree-Rangaroo Dendrolagus ursinus Togelkop Tren-kengaroo

zagorchesces hirsutus Rufous Hare-wallaby zagoserophus fasclatus. Banded Mare-Wallaby

[Macropus para Pare Walleby]

anychogalea fraenata Bridled Walltall Wallaby

anychogeze luneta crosent wailtall Wallaby

Instertrote

Erieceldee 
parnutes

(55) Cenridas app.

Thapelemer spp. Gontle 2omura

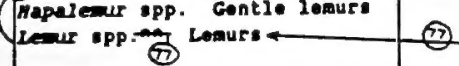

[Lepilemur' ipp. Sportive lemure]

(392)

$\rightarrow$ Colroseleldat spp.

[ulocebus spp. Halry-oared twarf lomur:

Cheirogaleus ipp. Fat-talled owarf lemurs

nicrocebus epp. Mouse lowur:

Phaser app. Pork-marked lenurs

(1820)

$\rightarrow$ Iodrildeo opp.

[indri opp. Indri:

Lichanozus epp. Hoolly leaurs

propt thecus ipp. Sif aka:

Daubeatonlldee

Daubentonia adagascartensis Aye-Aye

Locialdes

cellitbrleldae

(17)-allithrix jecchus aurite inite-oarad Marnoset

(i)-callithrix jecchus flaviceps Buff-heeded Marmoset

(17) teebuella pygmed PJEYy Marmonet]

Q Leantopl thecus spp. colden tanerlas

(it) Seguinus blcolor Bare-faced Tamerin

(1) Saguimus lewcopus volte-pooted Teanto

(1) Saguinus aedipus cottos-beeded ranerla

carilniconidat

callinto goeldil couldi's Marmot

cobldes

Nouted palliate Mantied Howler

iteles geoffroyl frantatus Bleck-browed splder Monkey

Ateles geoffroyl panamensis Rad Splder monkey orachyteles arachioldes Woolly Splder tonkey cacaj 10 spp. Uakarls

Chiropotes abinasus inite-nosed saki

Lagoehrix flaricauda rollow-talled Wooliy monkey

salde1 cerstodil contral merlean squirrel monkey

\section{Cercoplthecidae}

Carcocebus galeritus galeritus Tana Rivor Mangaboy

(1) Cercoplthecus drana Diana Guenon - - - - -

(1) colobuz pempanes1 k1rkt zanzibar rod colobus

Q colobus rufonitratus Iana alver colobus

Macaca slenus Llon-talled Hecague

132 masalis app? Pig-talled Langur and Proboscls

[masal1s larvatus probosels monkej]

(0) Pap10 leucophaeus Dr111

presbytis entellus Hanuan Langur

presbyt1s geel coldon Langur

(1) Presbyts plleata Capped Langur

(2) Presbyt1s potenziani Mentaval Langur

[Pygathrix opp. Douc/Snub-rix nemaeus Douc] Monkey

(

[S1alas cancolor Plg-talled Languri]

I

Colobus pementel gordonorve Uhabe led colobus]

colobus verus olive Colobus

macaca sylvanus Berbery teecque

[Presbyt1s fohnt1 Milglel Langur (19) -

Appendix II

pRImatBs app.* Priates finemur catta* Ring-talled Lomer]

$\left[\begin{array}{l}\text { Lor1s cardigradus slender Lorli } \\ \text { mycticebus coucang slow Lorts }\end{array}\right]$

[cebus capucinus ndto-faced capuchin]

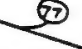


Appendix I

Appendix II

Pongldae

(77) Pongldae spP, Gorl11a, Orang-Uten, and chlmpanzees 4 [corllia gorlila Gorllia

Pango pygmaeus abel11 sumatran orang-Utan

pongo pygmaeus pygmaeus Bornoen Orang-uten

\section{EDrTata \\ Hy recopbagldae}

Aradypodide

Desypodidae

(1) Priodontes axtmus GIent Armadil10

Prowidore

renldae

mants teminck1 Tomalnck' Ground Pangoll

MACONORPH

Leporldee

Caprolagus hispldus HIspld Hare

panerolagus diazi Volcano Rabblt

CODEMTI

Seluridat

Cymanys mexicanus mexlcan Pralrie Dos

\section{veteronglae}

Duridas

tepor1llus cand1 tor Greter stlek-nest Rat

[Morays aquilo Northern Hoppling-House]

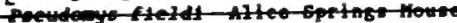

preudarys flueus sioky Mouse

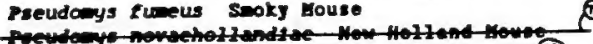

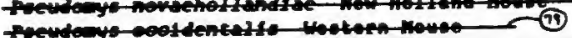

Paeudo ys pracomes Shark Bay Houso

Preudorys shortidged Hoath Hous of

xerays woldes False Water-Rat

syzons pedunculatus central Rock-Rat

$$
\text { Colpebl111dee }
$$

(7) Chinchilla spp. Chlnchliles (South American

Chinchilla spp. Chinchilias (South awerlean
popn)

Foinchilla brevicaudata bollviana Bollvian

Castoridee

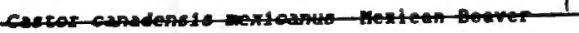

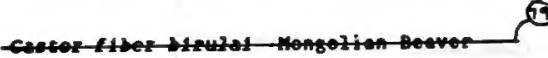

Myrmecophaga tridactyla clant Anteater Tanandua tetradactyla chapadensis Hato Grosso Collared Anteater

(4) aradypus variegatus srow-throeted sloth

Arvicolldat

Nesolagus netscher1 sunatran Rabblt

Larlscus hosel Four-strlped Ground squirrel Ratufa spp. Orlentel glant aguirrel:

Dipodanys ph1111ps11 phIIIIps11 Phllllps"

Kangeroo-Rat
Q

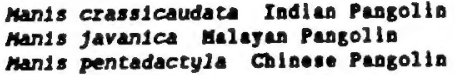

manls pentadactyla Chinese Peogolia

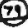

$\rightarrow$ Notonys epp. Hopplag-Hice 
Appendix I

Appendix II

CETACEA

(a) Pletenietidee

Iipotes vexil11fer inite Fles Dolphin 9. Platanista sp. Susus

(1) [Platanista gangetica Genges Susu] (ब)

platanista anor Indus susu

$21 \mathrm{pl} 11 \mathrm{de}$

serardius spp.

Hyperoodon epp.

Pbyoteridat
(4) Physecer macrocephalus sperm male

\section{Delphleides}

6 Socalis epp. Tucuxls

(69) Sousa epp. Hump-backed dolphins

\section{Phocoentale}

(9) Neophocsena phocaenoldes finless Porpolse (0) Phocoena sinus Cochlto

\section{Eechrlebtide*}

(1) Eschrichtius robustus Grej male

Balunopteridee

salaenoptera acucorostracak Hinke whele (all !

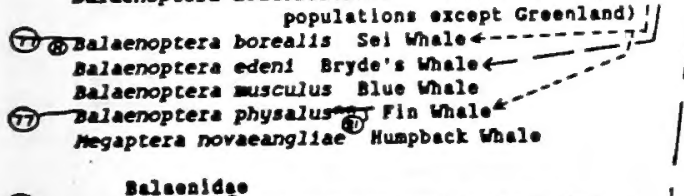

(ब)

1

CETACEA opp, * meles, dolphins and porpolses

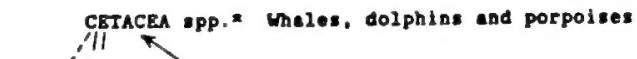

(3.) palaens spp. 9 Right weles und Bowhed whele

[Balaena pysticetus Bowbed thele]

copered marginata pygay Right whele

[rubalaena ipp. Rigbt welos]

cuerroes

Cendeae

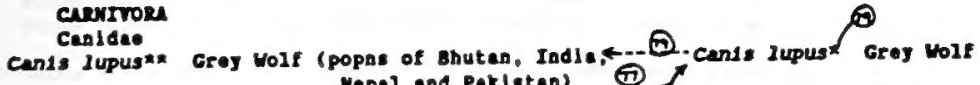

(1) toalaenoptera physaluge pis male]

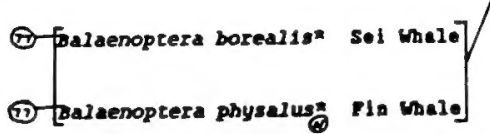

Can1s Iupus monstrabli1s Texen Grey Wolf]
6 Speothos venacicus but Dos

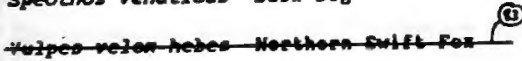
(7) Duslcyon griseug Argentine Grey Fox
(7) vulpes cana sienford" Fox
(S) rulpes zerda (1) Fonnec Fox

(4) Cuon alpinus Dbole

(0) Dusicyon culpaeus colpeo pox

Uraldae

Q7) Uriluropoda melanoleuca clant Panda Helarctos nalayanus Sun Bear 4

(A) Selenarctos thibetanus Asiatic Black Beer

C Selenarctos thibetanus gedrosianus Baluchisten

(7)

Tremarctos ornatus specteclod Bear Black Bear

Tremarctos ornatus specteclod bear

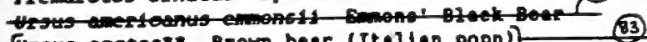

[Ursus arctos* Brown bear (Itellan popn)]

6 Ursus arctos 1 sabellinus Hivelayan Brown Bear ursus arctos nelsoni Mexican Grizzly Bear ursus arctos pruinosus Tiboten Brown Beer 


\section{Drocjonidee}

Hustelides
Alurus fulgens Loser Pende
(4) Aonyx conglcan. cameroon clawless otter (popns of Caneroon and Migerla)
Q

Conepatus humboldt11 Pategonlan Hog-nosed skunk

Enhydra jucris nereis Southern see otter

Luera tellna Harine Otter

OLuerd langlcaudis South American Rlver otter

zuera lutra Eurasien otter

wera provocax Southern Rlver otter

(2)

Lutednae spp.* otters

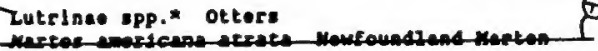

muscela nigripes Black-footed Forret

pteronura brasiliensis Glant otter

Viveridee

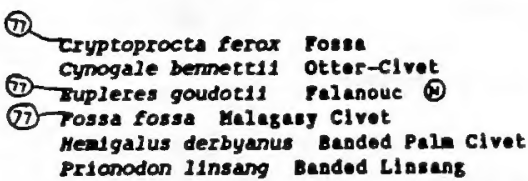

Prianodan pardicolor spotted Linsens

Hyealdae

Hyaena brunned Brown Hyaena

selldee

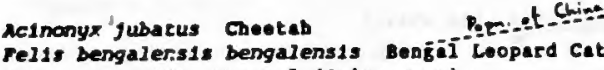

(4) Pelis caracala caracel (asian popn)

Pella concolor coryl Floride Pune

pells concolor costaricensis coste Ricen Pua

rel1a concolor cougar restern Puna

pel1s jacoblea Andean Cat

Pelis marmorata Harblod cat

relis nigripes bieck-footed cat

rel1s parda11s carns Coste Rlcen Ocelot

rel1s pardal1. atcis south Brazllian ocelot

relis planiceps riat-headed Cat

rel1s rublginosax* euty-spotted Cat (Indient

popn)

(1) Pel1a rufa escuinapae central Mexican bobcat

Pel1s teminck Aoletle colden cat

Pel1s c1grina anc111a Coste Ricen Little Spotted

Cat

Pel1s wed11 nicaraguae Contral Aerican Harsay

pelis wiedil sulvinia Guatealan Margay

Fel1s yagouaroundi cacont 11 Taneullpes

Jaguerundl

Pells yagouround fossata Guatemalen Jaguerund

Fel1s yagouaround1 panamensis Pancmenlan

Jaguarundl

Fel1s yagouaround tolteca sineioen Jeguerundl

weofells nebulasa clouded Leoperd

panthera leo persica aslatle Lion

panthers onca Jeguar

Panthera pardus Loopard

Panchera tigris*a Tiger

Panthera uncla snow Leoperd

Pelis cancolor ayensis Guetenelan Puse pel1s cancolor niseoulensts Hontane Puna

Fel1s lymx 1sabel11se Ilbeten lynx

re11: pardal1s* oeviot

\section{Fells serval sorval}

Fel1s elgrina* Littie spotted Cat

Pel1s wled11* Eargay

Pelts yagouaround1* Jaguarund

[Panthera leo persica asietic Lion]

Panthera tagris arace siberien Ifier 
PIMKIPEDIA

oterldoet

arcrocephalus tomsendl Guedelupe rur seal

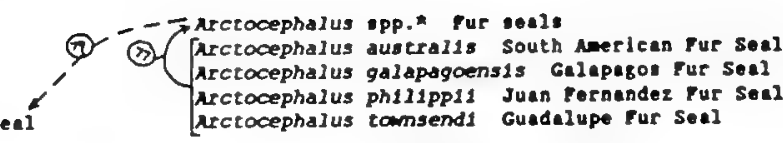

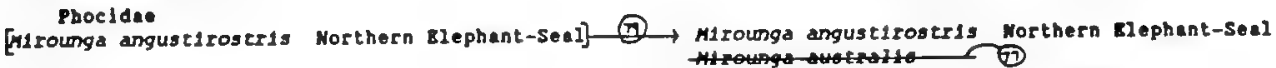
Hizounga leonina southern slephent-seal

manachus app. Monk seale

SUBULIDETIL

orjeteropodidee

Trososciore

Iephentidee

Elephas meximus Asien Elephant

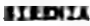

Dugongldee

Dugang dugon * Dugons

Itlebenidae

richechus inumgus Amezonlan rente

Trtchechus anstus Carlbbean medatoe

\section{perissonitr.}

Iquides

(3) zquis africanus Africen HIId An:

(0) Iquis grevyl Grev's zebra

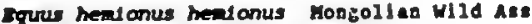

Equus heatanus thur Indian Wlid Ass

rgure przewalskil Preewalsh ' Horee

rquru rebra sebra Cape Bountain zebre

orycteropus afer Aerdvark

(7)

Laxodonta atricand Afrlean Mophant

Dugang dugon* Dugong (Australlen popn)

Trlchechus senegalensis Mfrican Banatee
(Q) Equus herianus* Asietlc wild as:

(20)

zquus zebra hargame Hertang's Kountain Zobra

250. Taptridee

65. Taplride spp.

paplrus balrdis contral recicen Iapla

raplrus indicus celejen tapir

raplrus plnchaque vountala Ieplr

Misocerotide

(1)

[Ceratotherito stan cottont Northern squere-1ipped Rhlnoceros

D1dernocertis subatraens1s Sunatran Rhinoceros

Fhlnoceros sondalcus Javan Rhinoceros

rhinoceros unicornis Indian Rhinoceros

Tap1rus terrestris srazllian Iapls 


\section{unIORACTH}

suldee

Dabyrousa babyrussa Bebleuse

sus salvanius Pyger Hog

Ilppopoteniese

$$
\text { Canclldae }
$$

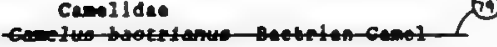

vicugra vicugra ticuna

\section{Cervides}

(7) Blastocerus dichotans Merah Deer

Cervus dand mesopotanicus Persilen Fallow Deer (Q)

cerne duravcell swap oeer

Cervus elaphus hanglu Rahalr Rod Doer

cervu eldi Thanlo

Q cerrus porcinus enanielcus Indochlnete Hot Deer

Q cervis porclnus calanianensis Calamban hog Deer

Qcervus porcinu kull Kuhl' Hog Deor

(5.) 1 ippocavelus spp. Duesul:

(IIPpocanelus antisensis Morth Andean Kuenu2]

Inippocanelu blewlous South Andean Huteul

Noschus spp at Hust deer (popns of Afghenleten,

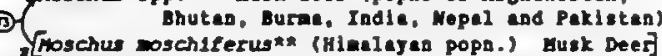

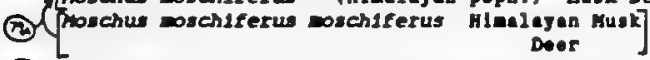

(5) Imelacus erinifrons black Buntjac

orotoceros bezanticus Papas Deer

pudu pudu soutbern Pudu

Boridee

Mdex nascaculatus Addex

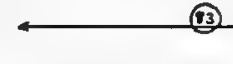

Intllocapta enerlcana penlnsularis Beje Pronghorn

intslacapra inericana nanoriensis sonoren

Blian bison athabescae vood Bleon

Propghore

Q Dos gaurus Gavr

Q Dos cutus Tak

Q Dos saure21 Toupray

Q Dublus depressicornis Lowlend Anoe

(O) Dubalus andorenals Teancau

(Q) Bubalus quarlesi rountein anos

Capra falconer1 chialtanensis chliten Markbor

capra falcaner1 jerdoni sulenen Herkhor

capra falconerl nogoceros Kabul Merthor

Capricornis suatraensis Malaland Serow

[analiscus dorcas dorcas Bonteboly

Zudorcas tericolor Taklo

Capra ralconer1" Warthor

(3)-Cephalophus dorals bey Dulker

(3) Cephalophus zebra sanded Dulker
Choerops1s I1beriensis Pyeng Hippopotums

Q2

Lana guanscoe Gueneco
Pudu Eeph1stophiles Morthern Pudu
Hoschus sp." Husk deer

[Addax nascanaculatus Idde]

fumotragus lervia serbery shoep

int1locapra avertcana wexicana Kexlens Pronghoro

(3) Garelle dana Dene Gazelle

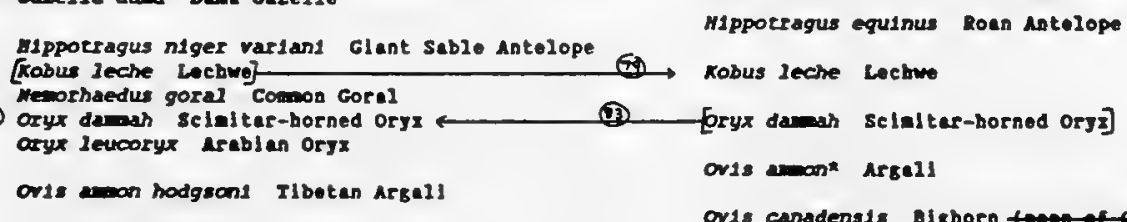

(- orls orlentalis ophlan Cyprus Mouflon

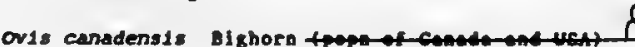

(B) Cephalophus fentinkd Jontiak' Dulker

Cephalophus ancicola Blue Dulker

13) Cephalophus og12bys Ogllby' Dulker

(45) Cephalophus sylvicultor Tellow-bseked Dulker

ovia vignel Uelal

pantholops hodgsont chiru

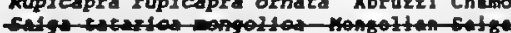

(2) [Paneholops hodgson! chiru] 


\section{Appendix I}

Appendix II

Ares
STRUTHIOKI rozacs
D Strutbiodicas
truch10 canelus ostrich (popns of Algerla, Burklne,
Caneroon. Central afelcen Republic,
Ched, Hell. Meurltenla, Moroceo.
Mtger, Miserle, Senegel and Sudan

\section{(7) enelda \\ 32. Thelda \\ rimeitropers \\ Inaldae}

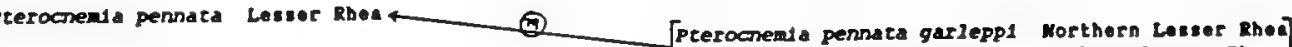

Pteroconemia pennata pensata Soutberb Laser ihes.

Rsea anericana albescens Argentlo Grenter the

Tinams sollcarlus Solitary Tinamou

SPHEXISCIPOR:ES

sphealecidae

(10)

Sphentscul huboidc1 Subboldt Paguin

noprcipzorfores

Podle l podides

Podilybus gigas Atitian Grebe

\section{Proctinintrones}

Dlanedelase

blanedea alberus short-talled Albatrose

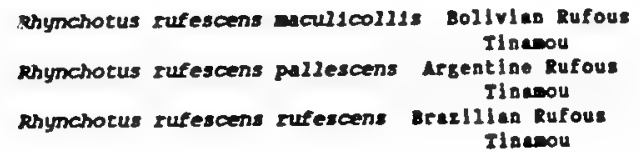

Ilsearou
Sphentscus desersus Jackass Pogulo

\section{pereunromes}

Peleens las

pelecanus crispus Delation Pollean

(3) [Pelecasus crispus DeInatian Pallea]

Sulldae

sula aboces Abbott" sooby

Fresetidee

Fregac andrews Christas Island Felgateblrd

\section{CICONIIForass}

cleontldee

cicanla clcania boyclana orlentel inite stork

3) pabiru gycteria Jablru

\section{Threskloralthide}

(O) ceranticus erent ta Northern Beld IDI: mipponsa nippon jepaneso Crested Ibls

Phonbleopteridee clconla nigra Bleck stork

Cerontlcus calvus southern Bald IDl:

Platalea leucorodia inlte spoonblil

Phoenlcopteridae spp. Flanlingos (83) Pphoentcoparrus andinu andeen rleslago Phoenlcoparrus jaesl jaee' Raniogo phoenlcopterus ruber chilensis Chllea Fialogo (19) Lhoenloopterus ruber ruber cerloben pleningo 
Appendix_I

\section{NISERIFOERES}

Anat Idae

Anas aucklandica nesiotis Caepbell Ialand Brown Teel

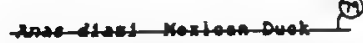

(4) Anas laysanens1s Laysan Duck inas oustalet1 Marlenes Duck

Eranta casadensis leucoparela Aleutlan Goose

Dranta sandvicens1s havalian Coose

calrina scuculaca mite-winged Wood Duck

Rhodonessa caryophyllaced P.0. Pink-hedod Duck

\section{Appendiz Is}

Anas aucklandiea auckiandica pueklend reland

Brom Tas

Anas aucklandica chlorotis New Zealend Brom Teal

Anas bernler1 Kadagencer Ioal

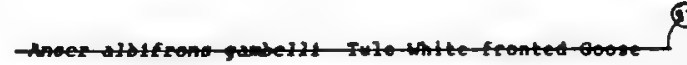

Dranta ruticoll1: Red-breasted Goose

Coscoroba coscorob. Coscorobs Swen

Q cygnus colloblanus jenkourkil Jankowakl's swan

cygnus melanocoryphus bleck-necked swan

(3) Dendrocygnd arborea Wet Indlan whlsting Duck

axyura leucocephala mite-hesded Duck

Sarkidlorn1s nelanotos Conb Duck

\section{PALCONI TORES}

Gymogyps callforntanus Callfornia Condor Vultur gryphus andean Condor

Aeelplteldee
Cethertldee

(9). Aquila helsaca Iaperial tegle

[Aquila hellaca adalberti Spanish Imperlel Eegle] (6) Chondrohiezax uncinatus wilsonil Cuben Hook-bliled rite

(1. Hallacetus alblcilla mite-tailed Eegle

Hallacetus alblclila groenlandicus Greenlend

(0. Hallaeecus levoocephalus Bald Bagle

[Hallaeetus leucocephalus leucocephalus southern

Marpla harpyja south Aerien Merpy Eagle

P1ehecophaga jefferyl Phillppine tegle

Pandionldee

Beld Eagle

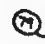

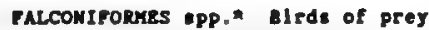

(exc. Cathertidse Pp. (New World vultures)

Acclplter genc1lis worthern coshewk 7f hociplter gundlachl Gundlech's Mawk (1) Acclpiter nisus Buralen Sperrowhawk (1) hegypius monachus claereous vulture

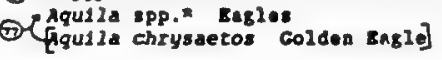

(17-Circaetus epp. Snake eagles

(1) Cizcus ipp. Herrlers

(7) Cypaetus batbatus Lenerseler

[Gypaetus barbatus mertdionalis african Lamergeler]

(77)-cyps fulvus Eurasian Griffon Vulture

(77. Hallacetus ipp.* Sea esios

7) Harpyopsis novaeguinede Mew Guinet Marpy Eagle (7)-HIVus mivus Red Kite

(1) Pandion hallaetus osprey

Falconidee sp. * Falcons and cerecaras

Felcoaldee

Falco araea sejehelles Kestrel
Falco jugger Legser Paleon

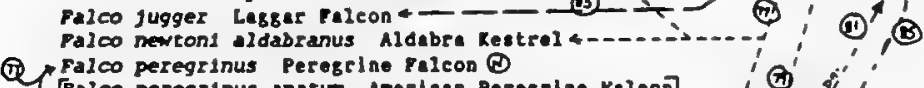

Falco peregrinus anatu anerlcan Peregrine relcoñ

Palco peregrinus babylonicus Arablan Peregrine

Felcon

Faico peregrinus peregrinus Europen Peregrine

Falco peregrinus tundrius tundre pero

Falco puncearus meurltus rostrolcererlne relcon], $;,$

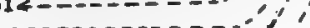

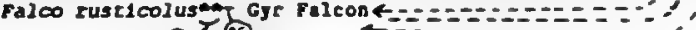

$$
\text { (1) }
$$


Appendix I

Meicinores

regepodildee

Macrocephajon maleo Maleo

\section{Crecidee}

(8) aburria jacutinga Black-fronted Plping Guen

(i) dourta plpile plpile TrInldad Plplog Gun

Crax blwnenbach11 Red-bllied Curastow

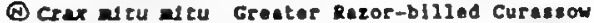
oreophasis derblanus Horned Cun

(1) Prenelope albipenis inite-wliged Guan
Append IX II

Megapodius freycinet abbottl abbott " Scrubtowl regapodius freycinet nicobartens1s Mlcobas

scrubfow

\section{Pardagldee}

cacreus wallichli cheer phesent collnus virginianus idgwayl Masked Bobwhlte crossoption crossoptilon mite Eered Pbesent

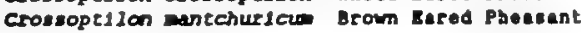

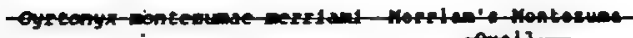
Q⿻日土龰?

ध

Lophophorus sp. Monals

Fophophorus impejenus Himalayan Mona?

Lophophorus lhuysil chinese Monal

Lophophorus sclacerl Scleter' Honal

Lophure ecourdst Bdwerds' Phessant

Lophure 1epertals Inperiel Pheasent

cophure swinhols swiphoe"s Pheesent

rolyplectron Ephame Palawen Peecock-Pbeesent

syraticus ell1ot rllot's Phesent

syratucus hude Hune Pheatent

syraticut elkado Mikado Pheasent

Tetrogalius casplus Caplen snoweock

reerogaliu cibecanus tibeten soowcock

jragopan blyen1s Blyth's Tragopan

Tregopan cabots Cabot's Iragopan

Tragopan wanocepheius Wotern Iragopan

rypanuchus cupldo eccracerl attwater" Pralele

Chlicken

Mrgusianus srous Creat Arsus phessant

Eatreus wallchil choer Phoasat]

cyrtonyx montezurve nearnst (exc. poph of USA Mexlcan Geerns" Montezune Quall

Cyrtonyx montezwave montezunse southern

contezune quell

Francolinus ochropectus Dflbout I Fraseolin Fraxcolinus mierstral swlerstra' Praneolin

Gllus somerat11 Groy Juaglofowl

tehagints cruentus nood Phoasant
(72)

Pavo wuticus Green Petowl

Polyplectron blculcuratu Groy Poncock-Pbeasat

Polyplectron gerneint Cormiln' Peacock-Pheasut

polyplectron alicente Maleyalan

Pescek-Pbesest

(7)

GTetrao slokosiendes ceucenlan Bleck Grouse

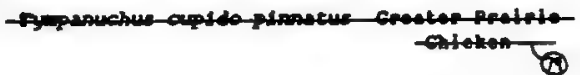


Append1: I

ceurroluess

Turnicidae

Pedloponldee

Gruldee

Grus americana whoopling crane

Grus canadens1s neslotes Cuban sandhill Crane

Grus canadensis pulla Misisippl sandhill Crane

crus Idponensis Red-crowned Crane

Grus leucogeranus slberien crane

Grus wonacha Hooded Crane

Grus nigricol11s Bleck-necked Crane

Grus viplo undte-naped Crane

Lell14ae

Trlchollmas sylvestris Lord Howe Wood Rall

Drpocbet Idee

Rhynochetas fubatus Ragu

otldidae

Chinydotis undwata Houbere eustard

noubaropsis beogalepsis

\section{(9) [chlanydoess undwaca noubare Bustard]}

52. cruldee app.* crane

(3) Finthropoides virgo Dowolmelle Crane

Balearlca regujorm southero Cromed Crane

Grus canadens1: pratensis Florlda SandhIII Crane]

Cald1raldus austelse hector1 Buff Woke 
Appendix I

Appendix IT

PSITTACIFORES

(1) Pelttucidee

C. Tnazona arauslaca Red-necked Amezon

(1) Amazona barbadens1s Yellow-shouldered Amezon

(1) amazona brastitensis Red-talled mezon

Q Amazon dufresniana rhadacorycha Red-crowned Amezon !

inezana guildingll st Vincent Amazon

andzont inperialis Imperial Amazon

donuzona Ieucocephala Cuban Amezon

Mrazona pretrel Red-spectecled mazon

Amazona verstcolor St Lucle Amezon

drazona vinacea Vlaeceous Amezon

imazona victaca Puerto Ricen Amezon

inodorhymchus glaucus p.e (D Giucous kece

Anodorhymehus learl Lear" Macaw _-

Ard anbigua Buffon's Hecaw $4-$

(1) Ard glaucoguiaris Blue-throted recawe

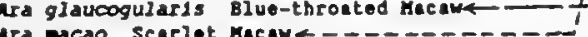

Ara rubrogenys Red-fronted Macaw $\longleftarrow$

Aracinga guarouba colden Conure

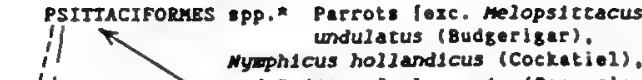

PSITIACIFORES epp.* Parrots lexc. Melopsictacus
undularus (8udgerigar).
myphlcus hollandlcus (Cockatiel)

myoplcus hollandlcus (Cocketiel).
if and Psitcacula kranerl (Rose-ringed

22 facatua cenutrostris siender-bliled Cockatoo

(1) Calyptorhymchus Iathan Glosey Cocketoo

coracopsis nigra barklyl sojebelles Vese Perrot.

(4) Cyanoliseus patagonus byront Greter Patagonian

Cyanopsited splxil Splx' Hacew

Cyanorampus auriceps forbesi forbes' Yellow-

fronted Paraket

Cjanoramphus novaezelandlae Red-fronted Parakets

ccopsletacus accidentalss pion. Might Parrot

Neophend chrysogaster orabo-bellied Parrot

[Weopheas aplendida scarlet-chested parrot]

Ognorhynchus 1cterots Yellow-aered Conure

(D) opopited dlophthalm coxent Coren's Doublo-yed

(i) Pezoporus willcus Ground garrot It Parrot

planopsitta plieica arazlilen Plieeted Parrot

Conure

Cyanoramphus marerb1 Orage-fronted Paraket

67. Eyanoramphus novaezelandiae Red-fronted Paraket]

Cyanorampus unicolor Antipodes Green Paraket

sumymphicus cornucus Morned Paraket

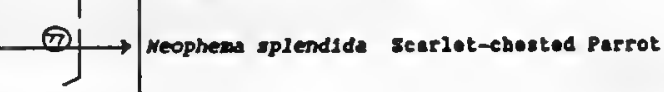

(10)

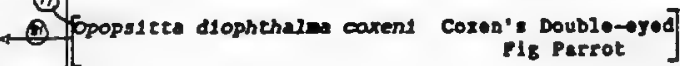

Prephotus ehrysopteryglus colden-shouldered Parrot

Psephotus pulcherrizus p. Paradise Parrot

psitcacule echo beurltius peraket

pistecus erithacus princeps Princlpe Grey Parrot

Pyrthura cruencaca Blu-throated Conure

Q Rhymehopsicta spp. Thick-bilied perrot

Rhynchopsicta pachyrhyncha Ihlek-bllled Parrot]

strigops habropelius Rakapo

Polcephalus rabustus Cape Parrot

(6) Polytel1: alexandree Princess perrot

Probosciger cterrimus Pain Cocktoo

prosopela persanaca rasked shining Parrot

(17)

Psephotus havarogater narethae littl.

Bluebonset

Tanygnathus Juclonensi: Blue-naped Parrot

cuculi rorates

Yusophas idae

Tauraco corytha1x Koyse Iureco

(4) rauraco porphyreolophus Purpl-crested Iuraco

stercipones

iytosides

(77) Iyto soumanel Hedagasear or

(C) Stchere blewitel porest oulet

(17) Hinax novaeseelandiae royana worfolk Inlend

Boobook owl

Minax squamipila natalis chrletmes Inlend

Boobook owl

Q otus gurneyl Mindaneo ow

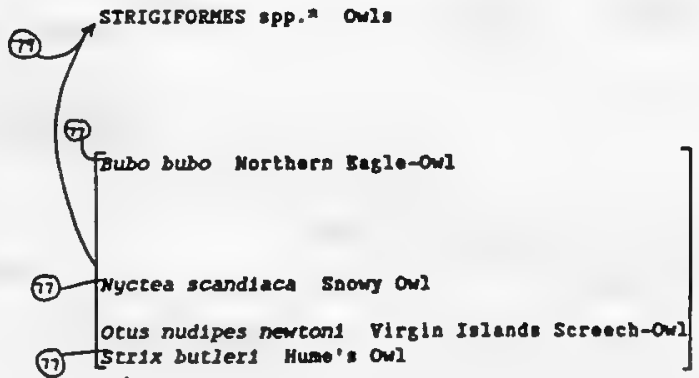


Appendix I

Appendix II

APODIrotades

Irochl11des

(6) Glauc1s dohm11 Hook-billed Hermit

Thoconiforases

Irogooldec

E. Pharadachrus nocino Resplendent Quetzel

(Pharamachrus nocinno costarlcensis southern

Rosplendent Quetzal

Pharanachrus mocinio mocinno Northern Resplendent

concriroledes

Bucerot 1dee Quetzel

\author{
suceros bicomis homral Worthern Great Hornbl11-- \\ Rhinoplax vig12 Heleeted Hornblil

\section{PICrrowes} \\ Pleldie \\ Crpephllus inperlalis Ioperlel Voodpecker \\ oryocopus javensis richards1 Irletran' inlte- \\ bellled Woodpecker
}

P1cus squanatus Clavirostris Wetero Scaly-bel11ed Hoodpecker

\section{pisserrom}

Cotlatidae

cotinga aculaca sended cotlage

Apholena cropurpurea Mite-wiged cotinga

\section{Pltidae}

pice rochl inlikered pitte

Aceros narcondand Merconden Horobl12

(1)--Duceros blcornis* Southern Greet hornbl11

Buceros hydzocorax hydrocorax cuzon Rufous

Horab111

suceros rhinoceros rhinaceros Kilejen ahlooceros Hoenb111

9. Rup1cols app. Coeks-of-the-Rock

[Ruplcola peruviana Andeas cock-of-the-rock

Ruplcola zuplcole culanen coek-of-tbe-Roek]

Pltta brachyura nypha Falrs Pltte

\section{Atejehoraltblate}

aerichorn1s clanosus Molsy serub-Bied

Elrundlalene

Preudochelidon irincarae mite-ejed River Martin

\section{ruselepidat}

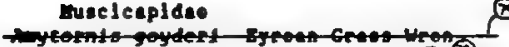

assyorn1s Drasdbent1 11 teoral1s p.o. Western

Rufous Bristiebird

Dasyornis langirosest: Watern Bristlablrd

Q M12tava ruecki Rueck' Blue Flyceteber

(2) Plcathartes ipp.

(Plcathartes gymocephalus inite-necked pleatherte

picathartes oreas Grey-necked Pleathartes

prophodes nigrogularis Wettern mipblrd

zosteroplde

zosterops alboguarls inlte-chested mite-eye

Hell phesidee

Neliphaga cassidix Helevet Honejeater

Fring 1111 de

(9) Carduelis cucullatus Red siskin

(4) carduel1s yarrel111 rollow-faced slatin

Eetri1didae

(1) bomblema oculaca Red-ared Firetell

D) Poephila cincta cincta Southern biack-throated

Fineh

Sturatide

Leucopsar rothsch1ldt Rothechlid'. Myn 
Appendix I

Ippendix II

Rept111.

TESTUDIMATA

Dereet and dise

(1) Dermatemys mans contral anorlcen River Iurtio

Exalda.

Bacagur baska Common antegur

ceoclewy hanlizonst Black Pond Iurtle

Clemmy muhlenberg1 Bos Iurtio

kachuga tecta tecta Indian Ient Iurtle

(2) Nanochelys tricarinata Throe-keeled Land Iurtie

morenta ocellats surmese suamp turtle

Terrapene coabuila Aquatic Box Iurtle

Ientudinlane

(2) Geochelane eiephanropus Galapago: Glant Iortolse

(1) Geochelone radiacs Radicted Tortolse

(1) Geochelone yniphoza madagescat Iortolice

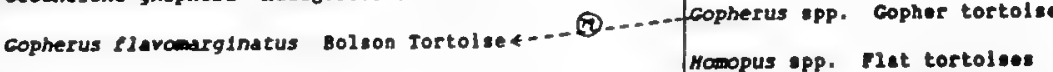

Kinizys app. Hinged tortolas

Malacochersus spp. Pancake tortolson

Q Psamposated geanetricus ceonetrle Tortolse

Pyxis epp. Spider tortolses

Testudo ipp. Pelceretle tortolsos]

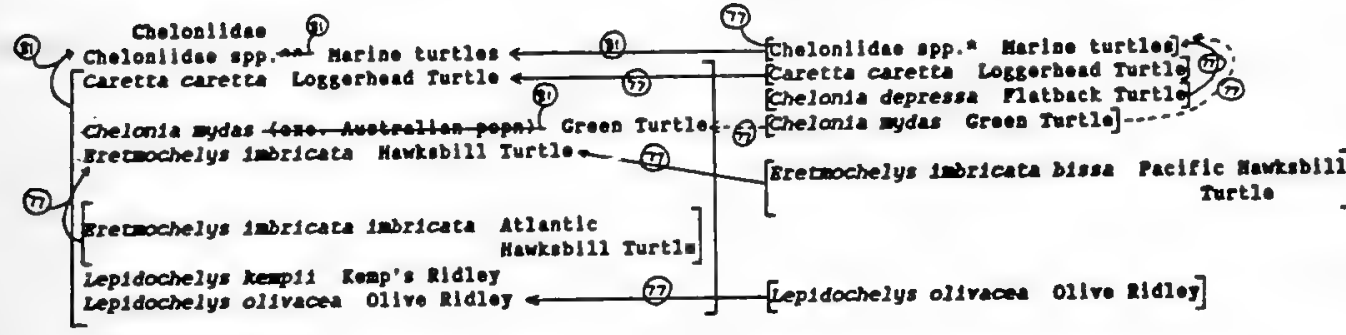

Darmoctelyldee

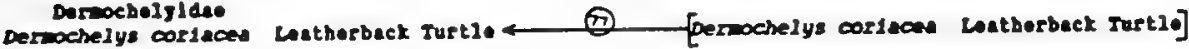

Ielongebldeo

Lisengs penctats puxceata Indlan Flap-ehell

$$
\text { Turti. }
$$

Trionyx ater slack soft-hell Turtio

Trionyx gangeticul Gases soft-ehell Turtlo

Tricony hure Peecock-ancked soft-she11 Iurtle

rriconyx nigricens Dark soft-shell Iurtle

Polemonduldes

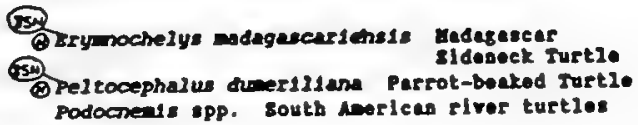

Cons1den

pseuderydura undina short-aocted Turtle 
Appondir II

Crocodruta

Aligatoridee

[Alligator alssissipplensis American Alligator] Alllgator sinensis chineso Alligator

Calman crocodilus apaporiensis Rlo Apaporla spectacied cainen

[calman crocodilus crocodilus south merlcan

spectecled calmen

calman crocodilus fuscus Brom spectecled cainen

Calman latirostris Broad-nosed Caimen Melanosuchus niger Bluck Caiman

calman crocodilus yacare Tacare

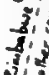

CROCODYLIA IPP,

(2) Ei:

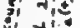

Palaeosuchus palpebrosus Dart Calesn

palaeosuchus trigonatus seooth-fronted calen

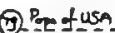

ti 5

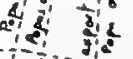

Crocodylidar (8) A crocodylus acutus Amériten Crocodile crocodylus cataphractus Sherp-nosed

crocodylus intermedius orinoco crocodlle

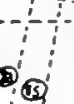

$-7 \frac{1}{1}=-2$

[Crocodylldee spp." crocodiles]

Crocodylus acutus American Crocodlle

Crocodylus Johnson! Johnson' Crocodile

crocodylus morelet1 horelet' Crocodilej crocodylus niloc1cus*tin Mlle Crocodile:=:crocodylus novaegutneae mindorensts Mindoro Crocod11. crocodulus porosugt crocodylus rhabifer

crocodylus slamensis slamese Crocodile

osteolaetus tetraspis Wost afrlch Dwarf Crocodile

Toulstans shlegel11 palse Gharlal

\section{Gerlelldee}

Gavlaits gangetscus Gherial

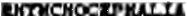

Spbeoodont late

Sphenodon punctacus Iutara

\section{gaver}

Cektooldae

Pygopodidee

Asendae

Chene Ieonldae

\section{Isuen 1 dae}

(11) Drachylophus epp. Fijl iguenes

cyclura spp, west Indian rock iguanes

(1)

Sauroalus varius Sen Esteben Islend Chuckwalie
(77)

(77) Cyrtodactylus serpensinsula serpent Island Gecko Phelsuns pp. Day seckos

(1) Paradelas orlentals quensland sneke-Lizard

(7) Uranastyx epp. Spiay-talied Ileerds

(95) Oradypodion spp, Dart ebaneleon:

(7) Chanaeleo spp. Chaveleon:

Amblyrhynchus cristatus Galapagos Herine Iguene

(2) Conolophus epp. Galepagos land iguenes

Cyclura sp. West Indien rock Iguanes]

Tguana epp. Green lguenes

Phrymosoma coronacu blalnvilled sen Dlego Horned Lieard

Cordy11dee

(1) cordylus spp. Giedled Ilzerds

(1) Pseudocordylus opp. Crag 112ards 
Appendis I

Iollake

\section{Eoloduratldeo}

\section{rerenlace}

Varanus bengalensis Bengal Monitor Varanus flavescens Yollow Monltor Varamus griseus Desert Monitor varanus kamodoens1s Romodo Dragon

\section{Appendix II}

Chentdophorus hyperyebrus orento-throated iniptal (7) Cracodllurus lacertinus Dragon Lliardet

(7) Dracaena gulanensis Calmen Lizerd

(7) Tuplnambis epP. Togus

Heloderma sp. Gila Mosster and lended Llzard

Varanus spp. Monltors

\section{maperres}

soldee

(1) Acrantoph1s mp. Hedegencer boes

(7) solyerla wrocarinata Round Ialand Bon 0

(77) Casared dussulet Roel-scaled Boe ()

Q2. Ipicrates inornatus Puerto Rican soe

[iplerates inomatus inomatus Puerto alean doe

eplarates wonensis tone Island $800, \ldots$

Iplcrates subflarus Javican Bo*

Python molurus nolurus Indien Rock Python

(1) Sanzinie edagascarlensis Hadagacer tree bo

colubrlate

aeplace

\section{(4) clelia clelis vusturane}

QCyclagras gigas Falie Cobre

Blachistodon westernami Indlen sgg-eatlog speke

(8) Thanophts couchl havand Mo-stiped Garter snake

65. Hoplocephulus bungaroldes Broed-headed Snake

\section{Aphible}

CAUDAZs

ingetcoldee

Anbystana duer1ils Acbogue

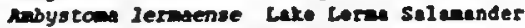

Amystand exicania Axolotl

Cryptobreacblaae

6 - indrias sp. clant valaendera 8

dindrias davidianus cbinene Glent selenender

andrlas jeponicus Japenese Glant salmander]

\section{Irues}

sufosides

atelopus varius zecerd colden Fros

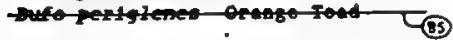

bufo superclliarls Caveroon Iond

Mectophrynoldes epp. Viviparou African toads

\section{Hobetrachidee}

(3)- Pheobatrachus epP. Pletypus Frog/Gestricbroodlng Frog

rasides

(85)-Rana hexadaccyia six-fingered frot

(85) Rana tigerina Indla sulifrog 
Pleces

cheroorromes

ceretodldes

Meoceratodus forsterl Australles Lugfish

comacurtar Fomes

colicantbides

Laelmerla chalumae Coelecanth

\section{CCIPENSERTRER:}

Acl ponserjdee

Actpenser brevirostru shortnose sturgeon

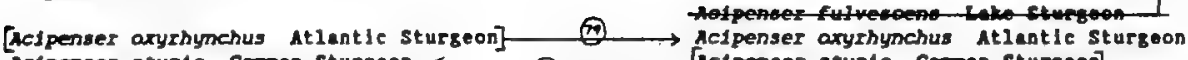

Acipenser sturto common sturgeon $\longleftarrow$ (93) [Acipenser sturio Comon sturgeon]

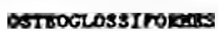

Osteoglosidee

scleropages formosus Asian Bony-tongue

Arapaima glgas Pirerucu

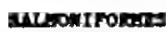

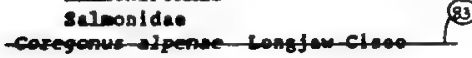

\section{Grentrorims}

Coprialdee

Probarbus fullent Ikan Tomoleh

Cetontandae

andalses cujue cul vi

steverroleas

schllbeldae

pangasianodon glgas cient catfish

\section{Amingriogin}

Grprinodont Idae

Poec1111dee

Faciforatis

Percidae

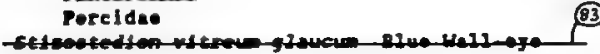

Seleonldae

Cynoscion macdonaldi Iotorb.
Salmo chrysogaster Herlean Colden Irout Stenodus levclchthys leuclchthys Beloribitsa

\section{(8)}

Caecobarbus geerts Afrleen slind Burb Fish plagopterus ergentiselive Wound in

Ptychochellus luctus Colorado River squarfish

\begin{abstract}
Cynoleblas consencise Pearifleb
Cymoleblas armoratus Glager Peariflsh

Cymolebias ainimus Haute Pearlfich

cymoleblas opulescens Opaloncedt Pearlfieb

cymolebias eplendens splesdld pearifieb
\end{abstract}

xlphophorus couchianus Loterey Platyfish

Insecte

LPIDOPTER

Peplilooldae

ornithoptera spp. Biraing butterfiles (4)

67) Frnithoptera lexandrae Quest Mlexandra's Braning

(17) ornt thoptera allotel Abbe Allotte' Birduing

(77) fornt thoptera chinaera chiseve Birtuing

(7) Omithoptera gallath collath birdulng

(i) fornithoptera meridianelis

(7) - ornithoptero paradised Paradlae Birching

(77) fornithopter victorise Quev Fictorle' Biraming

(7)- CPanasisus apollo nountein apollo

[Parmassius apol10 apol10 turopea rountaln Apol10]

(9a) Trogonoptera sp. BIrduing butterfiles (i)

(त)

Troides app. Birdwias buttarfles 0 
Arachnide

ARUARR

Therephoside

nolluees

mizora 0

ajtillate

\section{Tearpotan}

Irldeculdat

UNIONOIan (D)

Unjonldee

conradilla caelata Birdwing Pearly Mussol

Drans droeas Dromedary Peerly Hussel

(O) Eploblasma curtisl Curtls" Pearly Musel

Q splobiasma florentina Yellow-blosmom Peerly Kuscol

(2) Iploblasea sapsonl Sampson's Pearly Musel

Q Eploblasas sulcara perobilgua mite Catopar Muteol

Q iploblasma torulosa gubernaculim Gren-blosem

$$
\text { Peerly Musel }
$$

Q Iptablesa torulose corulosa tubercled-blosen

(6) Iptoblasm turgidula Iurgla-blossos Pearly Mussel

(4) Tploblasma walker1 Brown-blossom Poerly Mulsel

rieconala cuneolus Pine-rayed Plgto Peerly Kused

pueconale edgariana shinj Plgtoo Pearly Museel

Laps121s higgins1 Histins" oye Penty Muscel

Lapal1s orbiculata orblculata Pink Hucket

Peerly Mussel

Laps121s satura Plein Pocketbook Pearly Munsel zapsilis virescens Mlabane Lamp Peacly Mutuel

plethobasus elcaericosus mite Werty-beck Peerly

plechobasus cooperianus orange-footed Plaplebick

tusel

Pleurobea plemt Rough Pigtoe Pearly Husel

(1) Potanilus capax rat Pocketbook Pearly Musel

guadrula incernedia Cumberland Lonkey-face Peerly

cusel

vusel

Q Tarolasea cylindrella Pele Lilliput Peerly Mused

() Unto nickliniand Kleklin' Pearly Husel

(Q) Unio canpicoensis reconatensis rasplco poarly

Fussel

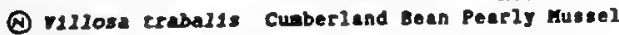

(15) Drachypela soleht Mezicen Rea-tneed Itrantule

(8) Choranyt11us charus choro

(2) Trldacnidae spp, Giant claea

8) [Frldacha derasa southera cleat cleo] (3) Tridaco glgas clat Cla

Cyprogen1a abere1 zalble Pearly Musel

Q Eploblasma torulosa ranglana ran-blosto Pearly kussel

rusconala subrotunda Loss sol1d wuseel Lamp111s srevicula Ozark Lanp Peerly Eused

Lexingtonia dolabelloldes sleb-jided Pearly Gusel

Pleurobea clava Club Poarly Musel 
STromstropros

canentide

Persphant Idee

mosonstropoda $Q$ bydrobildae
Q Papuseyla pulcherrias Manus Green Iroe sall

Paryphante spp. (All Now Zealeod app.) Abber onells

\section{Antbozon}

ArtPAтRAT

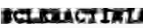

Poellloporidee

Aeroporldae

Aserleldee

ronslide

Fevlidae

Morullaldeo

Evesidec

Pectiolidae

Carjophy 111 Idee

\section{Iydrozoe}

Mranciy

III eporidee

\section{Nejonerle}

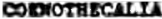

nel loporidae

STOLONIFER

Tubiporidae
(E) XurIPATHARIA epp. BIeck coral.

(6) Poc1llopora epp. Brom ste eluter corals (1) Serjacopora epp. sirde rest corele (5) Stylophora spp. caullelower coral.

(3) - acropore epp. wraneh corals

(6) Pavona pp. Cactus coral:

(5) Tungla sp. Mushrou corals (3) Halcal tra spp.

(15) Polyphyl114 opp. Feather corels

(5) Pavia opp. Draín coral.

65 platygyra spp. Braln corals

(35) werulina upp. Heruline coral.

65) Lobophyl11a spp. Arain root corals

(65) Pectinia spp. Lettuce coral.

(85) Luphy111a epp. Braln trumpt coral:

(85) H11lepora IPP. Wello flro coral.

(85)-Hellopora opp. Blue corsls

(65) rubipora spp. Organplpe corals 


\section{B Marices:}

(13) Agave artzonlca New Rlver Ageve

Agare parviflora Llttie Princese Ageve

(63) Agave victorlae-reginse Quen Ageve

(ai) Wolina Interrata Dobesa Bestras:

ipoctractus

Pachypodilo nanguanum \&

Pachypadil app. Mephent" trunk

\section{maceat}

Mocasia aderane

Nocas1. rebrina

\section{Mndscres}

(O) Panax quinquefolius Anerices Giaseng

mucserscest

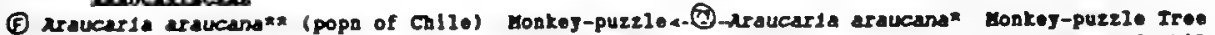
Tres

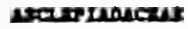

Q Ceropegia spp. rosery vises (7) Preree indica

\section{matmat}

\section{(10) byb11s spp. sybllse:}

croveras

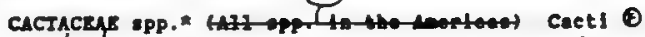
ancistrocetus tobusch11 Tobusch' Flshbook cactus Arlocurpus avioldes Imeullpse Liviag-rock Cactuse-

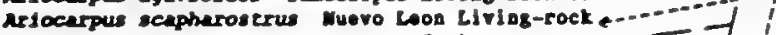

Cectu:

Mricenpus erigonuse- - -

Artekiu ritterl istoc Cactus - -

21

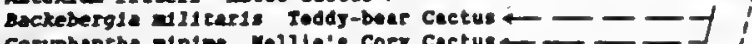

Q Coryphenthe ainia Mell1": Cory cectus

0 coryphentha inead11 sneed's Pincushion cactul $\leftarrow--1$

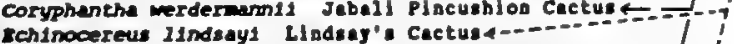

Oschinanstu erectocentrus

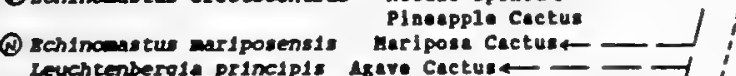

robesta principls Agave Cactust- - -

cdougallil IncDougal' Cactus - _ - -

nallilaria pectinitera - - - - - - - i

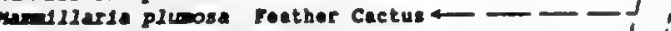

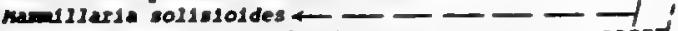

Obregon14 denegr11 irtlchoke Cectust-...-

Pediocercus bradyl Brady' plocushlon Cactuse-

Pediocactu despainit Despein' Cectur

Pedicactus lonciton11 Cnowlton's Cactur--

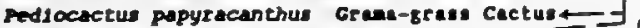

Pediocactu paradinel Peradine' cactur $\leftarrow$

Pedfocactu peebletianu Peobles Mavejo Cactur

Pediocactus olierl silor' Placushion Cactur-

6. Pediccectur mint

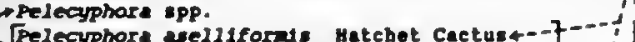

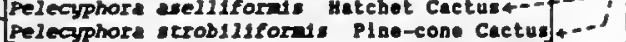

sclerocactus glevicu Uinte Beil hooklese Cacth

Crhlpsalts

.

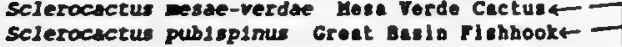
Cectus

sclerocactus wightias Wright" Mbhook Cactus

strombocactus disctforms bisk Cactust- -

rurbintcerpus app. Turblalearpus cectl- - -

M11coxia cerool111 Lab'-tell cactuk- 


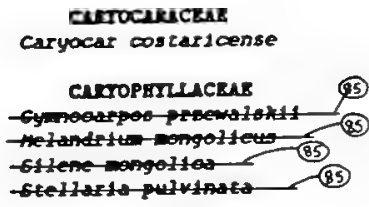

(3) Dudleya stolonifera Lagune Besch Dudleja

(3) Dudleya eraskiae Santa Berbare Ioland Dudleje

\section{artisucus}

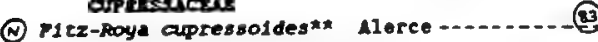
P1lgerodendran uniferim

cenmucres

cresuctes

Dreperstetes

Dressonincens

Drormuces

Drosconeacer

menceres

arreoknuas

racicese

\section{rougurrences}

(13) Pouguteria fasciculeta

(3) Pouquieria purpusil

\section{corruncris}

Prepusa hookeriana senrlet-flowered Prepusa $\rightarrow$ FItz-ROYa Cupressoldes" Llerce (coutel popa

(2) Crathenckar spp. Tree foras (9)

Cyathed capensis]

cyathea mexicans

Eyathea saivinit

(7)

Crchorcert spp. cjeads (1)

(63) Short18 galac1fol1s oconu-solls

(1) Dtcrsomiacrar spp.

DIDIRREACER spp.

(1) Dloscored deltolder

(3) xainda cuneate inite wicky

ruphorb1a ipp. (axe. epeeles mieb are dot oneeuleat) Euphorbla:

(1) Quercus copeyents copey ouk

(3) Pouguleria columaris soojul Irot 
Appendix I

mosodrecus

marrenacese

rastaned Darbouri1

JUEIUTHCER

sngelhardts pterocarpa

LECU:TMOSA

Amoptptasther- 85

Cyname tra heni tamophylla

platymiscilo plelostachyom

rachigalla versicolor

\section{LHIAcen:}

Hoe alb1da

Noe plilansis

Noe polyphylla spiral Aloo

Noe thomeraft11

Noe rossli

mertanticens

Lavolsiera Itanbesa

mucur

curea longlpetiols

\section{erscan}

antocarpus costartcensis

erracter

(1) Exparmacerer

(1) Tepenthes ragah Kinabulu PItcherplant

\section{oncuraceres}

Catcleya akinmerd Wite Mun Orchlo

Catueya triasse Corlotan Orcbld

Didicles cuminghadi

Lelia janghersa

Lel1s labera

(10) Lycasce virginsls: var. Albe

perlaterid elata Holy Chost Oredid

penenthers inchootiana Rod Vande orchlo

vand coerulea Blue vande orchlat.....

raver

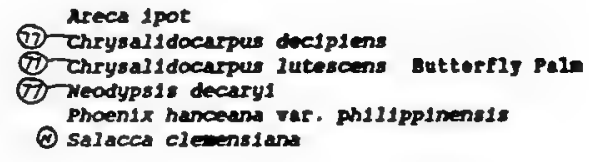

Appendir II
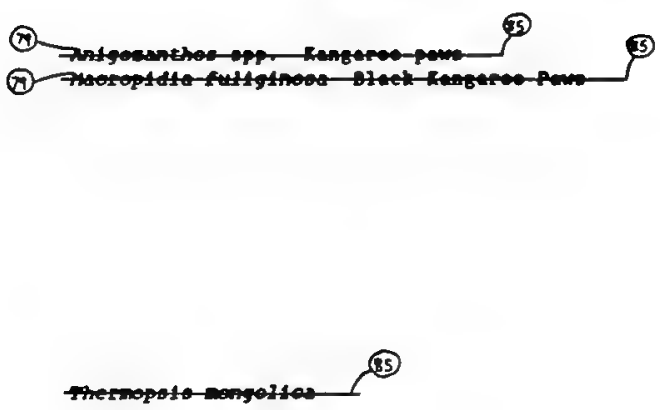

(E) Noe Epp. N1008
(9) sorletenta hulls: Mexlean Mabogay

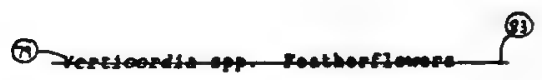

(6) prChIDACRAR app.* orchlds (2)

$\mathfrak{6}^{\prime}$

Princans

As1es guatemalensis Gutemelen Fit

Wter nebrodento- (63) 
Appendix I

podocinpaczer

Podocarpus costalls

podocarpus pardatorel Perlatore' Podocarp

Porvucicher

putoureris

prosucers

Orothanus zeyherl Warsh Rose Proted Proted odorata

\section{wivion}

salned etormae Ayugue

arucur

Meruontacer

(1)

Sarracenta abarensts Labamessts Alabuea

Cenobrake Pltcherplent

(1) Sarrecens jonesil kountaln swet Pltcherplant

(11) Sarracenia oreophila Green Pitcherplant

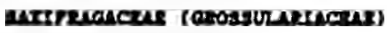

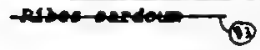

solunctas

remarencer

ODStangeria erlopus nottentot's Hod

irtacuractur

Totrax

monerancer

ormere

celett-dis

rearacus

virrscaracke

(i) Mejutechia bainesit

2urracen

Welwitseble$$
\text { zurtaces: }
$$

Ceratozanta sP.

ancephalartos spp. Bread-Palas

microcycas calocoma Palne Corcho

\section{zinctarencin}

uedychiw ph111pplnense Ginger LIIy

\section{moopmulate}

Appendix II

Anacamperos spp.

(3) Lewisia cotyledon siek rou Lewlite

B) Lewisia magurel Meguire Levile

(3) Lewista serrata sav-toothed Lowleila

(3) Lewisia needy1 Tredy' Lailsia

Cyclawen tpp. Cyclasent

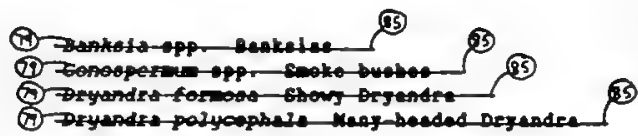

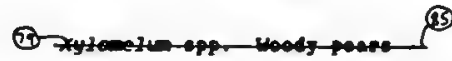

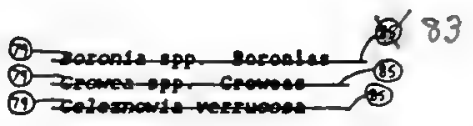

(2) Darlingtante cellfornics cellforale Pltcherplent

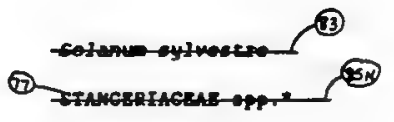

(i) Pterygota excelsa (1)

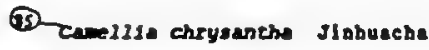

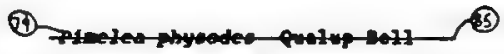

(15)

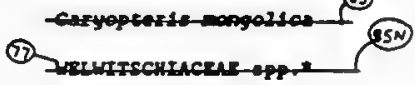

Q2)

-zarracens epp.* zalae (6) 


\section{Appendix III - at 13 November 1986 and Incorporating enendment made ince \\ the Convention came into effect}

\section{INTERPRETATION:}

2. Specles included in this eppendla ero reforred to:

a by the name of the specles; or

b. a betag 11 of the epecles Included in a highar taxon or deslgneted pert thereof.

2. The abreviation "ipp." Is used to denote 11 pecles of a hisher texon.

3. Other references to texe higher then pecies aco for the purpose of Information or elaselfication on 13 .

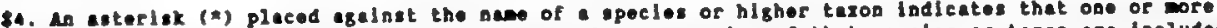
ceographiceliy seperate populatione. subsecles or epeclos of thet pocies or taron are included lo Appendix $I$ and thet these populetions, subsecles or species are excluded from Appendiz III.

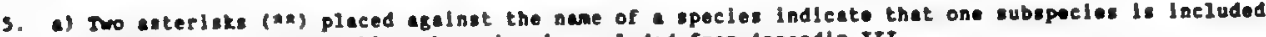
10 Appedis II and that this subsecles it excluded from Appendix III.

b) Tro aterlaks (*R) placed egelnet the name of apecles or bigber taron ladleate trat one or are

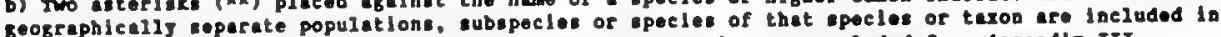
appendi II and that these populations, subpecles or species are excluded fron ippondiz III.

6. The nave of the countries placed ageinst the nases of specles or other taze ere those of the Partles sublttiog these epecies or tax for inclusion In this appendix.

7. Aaj enlas or plent, whether ilive or dead, of epecies or other taron Ilsted in this appendix, is covered by the provilions of the Convention, as is any readily recogalzeble part or derlvative thereof.

8. The sybol (C) pleced agalnst the nane of apecles or higher taxon denotes that this specles or algher taxon Is Included en part of appendix I. except for canada mich ontered e epelfle reservation with regard to then.

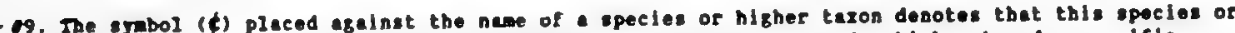
hisher tazon is Inciuded is pert of Appendix II. except for Canede wich ontered apeclfic reservation with regard to then

10. The smbol (M) Indicates that further dotall concerning that taros may be found in the wotes on peges 30-33

- These clauses have been inspplicable since 4 Pebruary 1977 or 3 April 1977.

\$ These clauses have boen Inapplicable elace 3 karch 1986

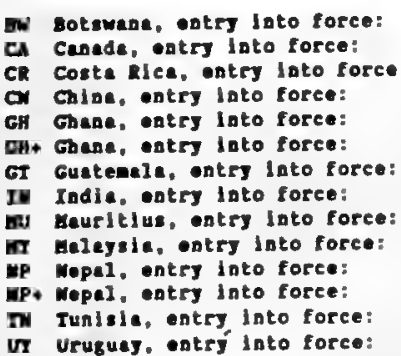

24 Apr $12 \quad 1978$ 16 Novenber 1975

28 october 1976 6 Decenber 1983 26 February 1976 5 Mercb 1986 23 Apr 111981 23 January 1984 4 December 1975

13 Norember 1986 16 November 1975 5 March 1986

22 Apr 111976 14 July 1976

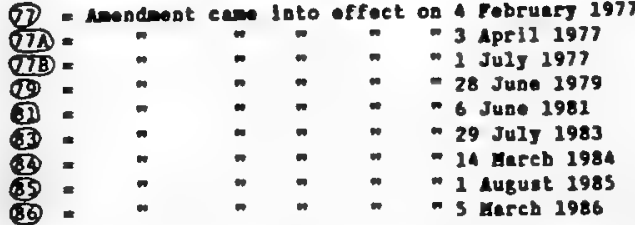

* Nomencletural chagge reaultleg trom Con. 4.16

- 1 - Upgradiag to Appendz I or II.

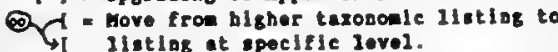


AppendlI III

Appendl I III

FA U M 4

Mane11.

(770)

Insectrore

Erlaceldev

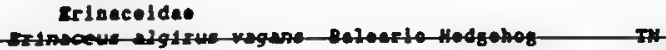

carroptres

(7T) Dhisolophldee

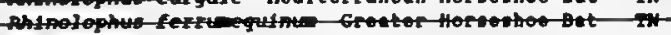

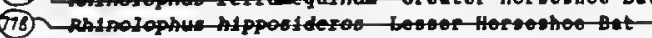

rayllostonidae

Vapyrops ineatus indte-11ned Bat

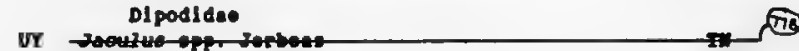

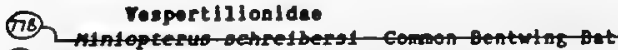

Myetrieldae

trix app. Crested porcuplaes

(76) plptotrelfus-ope plptatrollos-

TH-

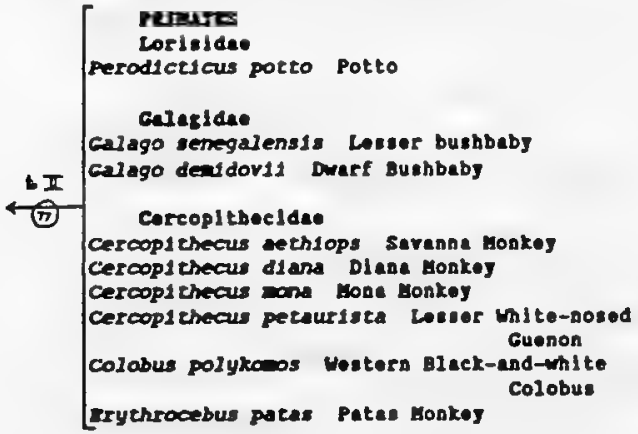

$\mathrm{CH}$

[n

Anoneluridae

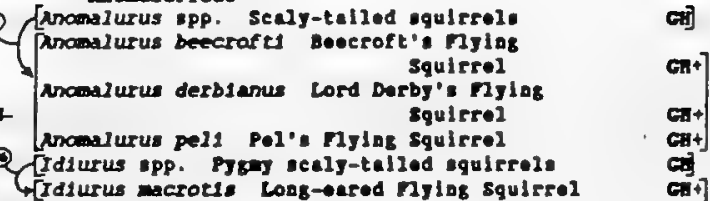

GIrldae

Poreuplae
Ports actican Crest

G.t] $\rightarrow$ (7)

Sphiggurus spinosus splas Iree Porcuplat (6) ir

Ctenodectridee

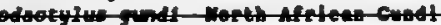

CrTack

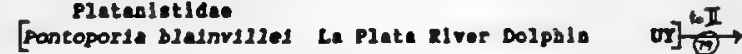

ropodont des

Eranodon anoceros vermel

a) $\stackrel{5}{\rightarrow}$

[sachrlehtius rabustu c cres ibere cis

Corret

yrimecophasldee

(0) renandua cecradectylan collered anteater

G

Dulesopteride

ralenopter ouculue c sue male

alaenopter physalus pis male

megaptera noveenglia c mupbek ibale

c. 1.1

प्रा

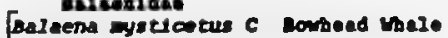

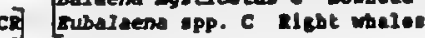

a) $\frac{1}{2}$

का [sradypu griseu Groy Throe-tood sloth

Cholopldae

choloepus hothaml Moffaen" no-toed sloth

Daspodide

Cabassous central1s Morthern Naked-telled Arned 1110

(D) cabsesour tarouny Grenter Maked-tailed Araed 1110

Proktrote

ranlace

Menis giganted Glant Pengolin

(Q) Mants tetradactyla Long-talled Pangolin

mande cricusps Ireo Pangolin

nopertu

selurldae

oplxerus eb11 Tominck's Giant squirrel

sclurus deppel Deppe' sgulriel

\section{citriver}

CR canjane

[rupes zerda Ponnec Fox

Ureldee

CR CA1 luropoda elanoleuca Glent Pande Uraus artelnu \& Poler Bear

III

Procjondece

Basgarlcyon gabb11 susb-telled ollago

Bassariscus sutehrast centeal inelces Caconistie

GH Nasua nasua sol1tar1a soutb Brapil1en Cot?

I.

a. 1

a)

c.

C2.

or

Laseldan

[Aonyx capensss afrlean Clamios otter

Calletis vitcaca Greater Grisos (D)

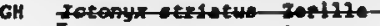

CR Eutra lutra Eurailes Otter

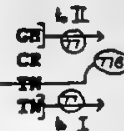


Appendi I III

Appendle III

(74) Butelldae (contloued)

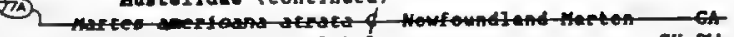
Nellivora capensis Retel GH,BH

(77) neillvia capensis patei

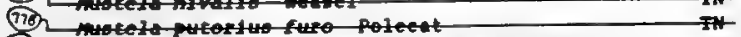

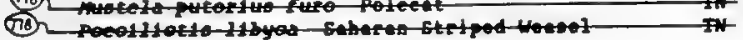

\section{Vitorrldae}

(2) civetuctis civetta afrlcen ciret

Protel1dee

Proteles criscacus sardwolf

\section{Greenlase}

(73)

Hywens hyems - stelpod-Hgeono-

\section{rollare}

Felds aurata arrlcen colden cat

Fel1s concolor cougar c Bestern Puse

tII Feis pardine perdine Lynx

(T) rel1s argarita sand cat

Fel1s alivescris I1byce worthern Afrles

vild cat

Fel1s ilveseris ocreats thbloplan HIId Cat

panebra leok clon

\section{PIntron}

adobenldev

odaberus rosearus welrus

\section{pososcros}

II Mephentidee

4 [L Laxadance africena Afrles Iophent

\section{Arropsone}

\section{Tayasseldae}

Tayassu rajecu Coliered Peccery

\section{Ijppopotenldee}

nippopotans aphiblus ilppopotanus

Iragulldee

myenschus aquaticus weter Chevrotaln

cervides

Cervus elaphus Dardarus Berbary Red Deer

Mran aricana ceralna Guetemalen Red

Drocket

odocolleus virginianus ayens1s Guatemalan Mite-talled Deer

6 II sovidee

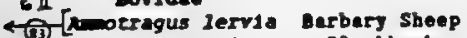

(i) intilope cervicapra Blackbuck

4 [Dison bisan achabasce C Wood siton

soocercus euryceros bongo (1)

bubalu bubalis Uater suffalo

Danallscus Iumatus Tressebl

carella curler1 ronl Gazelle

(7) Cuzella dorcas Dorces Gerell.

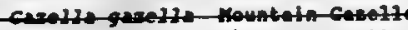

5 II Grella Jeptoceros" send Gezelle
[HIpporagus equinus Ron intelope

recracerus guadricormis Four-horned Antelope Tragelaphus spekel sitatunge (0)

cri]

GI

CH

IN

GH]

MP

GH
Areat

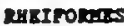 \\ pholdee}

Rhed acricanativ Greator thee

\section{croonfrovizs}

Ardeldae

Bu Ardea gollath collatb herog CH

Dubulcus ib1s Catt10 Egrot (9) CII

Casmerodius abu Grost igret (1) GI

BW Egretra garzere Litti sert

Cleonlidee

Iphlpplorhynchus senegalens1s saddloblll stork G. Leptoptilos crumentferus arabou stork

c.

Treaklorndthldate

(770)

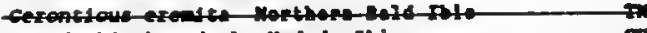

Magedashia hagedash Hedede Ible

Lapribis rara spot-breated Ible

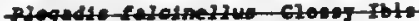

mresklomis aetoplcus sacrod Ibl:

Poonicopteridae

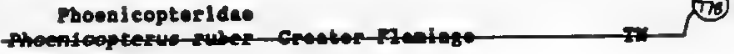

\section{Arsterror.cos}

ch Inetide

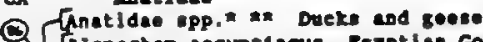

flopochen agyptiacu Egjption coose

Anas acued Morthora Platell

Juas capensis capo Toel

Anas clypeata wortbern sboveler (2)

hra: crecos Conos Toel

Anas penelope Iurasian Uigmon

guerquedula Gargene

Aythya nyroca Ferrugloou Duek $\Theta$

bendrocygna blcolor Fulroue inlatiling Duck $\theta$

bendracygna vidure ulte-faced uniatling

Duek

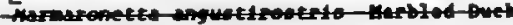

- Fetcapus auritus africen Pygur coose

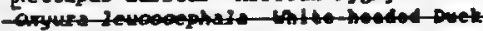

[Plectropterus gambensis Spur-ulaged coose

IN Preronetra hartiaubil Gartiaub' Duck (C)

at

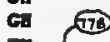

in-

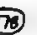

Irconiroteras

Pandlosdde

[Pandion hallaetua Oeprey

c. C. In $\stackrel{\hbar \pi}{\rightarrow}$

seciplteidue

Ti] [Aeclpitrides spp. Devte, eaglon (coste lee

MP [

cA] [Aquila epp. regles

GH(1) Aquila chryseczos \& colden Ragle

MP, - tul Cypaetus barbatus Lemorgeler

GH Gyps fulvus turasien Griffon vulture

IN Fallacetus Ieucocephelus Ilescanss Worthers

IN [

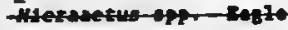

SPP.)

sagittarlides

Eagittarius serpentarius secretary Blrd

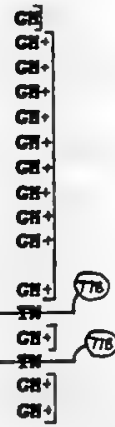

Falcondane

Falconldee spp \& raleons

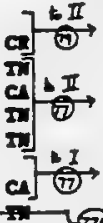

478

ㅂ. $\stackrel{6 \pi}{\longrightarrow}$

ली 
AppendIr III

\section{Gallrobers}

Craciase

ortalis vetula Plain Chachelece

Penelopina nigra Hlghland Guan

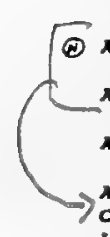

Paslealdee

arborophila brumneopectus Ber-becked Pacteldge

Agelastes meleagrides inito-branted Partrldso
paested
inenf owl

Agriocharis ocellata Oeelleted Iurkey

Caloperdix ocules Forrugl nouk Wood-Partridge Lophura erythrophehalo crestless risoback Pheesent

Lophur Ignst Crested Firobeck Phesestent Melanoperdix nigra Black Wood-Parteldgo polyplectron inopinatil mountelo PeacockPheseant

Phelpartis ocellata Crosted Argus Phemant Rhizothera longlrostris Long-billed Parteldge Rollulus roulow Crested Wood-Parteld ge

Tragopan sacyra Satyr Iragopan crax rubra Grest Curassow arboroph1l charitonil Cheatnut-neckleced
Partrldse

Appendis III

\section{PSTrictromes}

Palteclane

CR, GT Poltecloes epp. Perrota

GT Tra ambigua Buffon' Hecew

CT Ara macao scarlet macau

Psittacula kramer1 Roee-rlaged Paraket

G교 II

$\underset{C H}{C R}$

\section{cuculiromes}

mX truophesides

Q [Husophesiden spp, nn Turacon

mY forytheola cristaca Great Blue Iuraco

Crinifer piscaror Uesters Gror plenteldouter

GH Musophaga violaces Vlolet Turaco

GT Tauraco acrorhymahue Created Tureeo

\section{Muraroms}

Tytondace

m

Trtonidat spp. Bars owls

strlsides

[strigidae ipp. onls

Subo bubo Northern Ragio-ow

Nycted scandiaca srowy ow

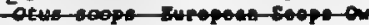

[strix nebulose Great Grey ow]

이

เII

G.] 6

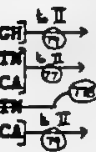

PICIroperes

Rephest Ideo

Rasphastos sulfuratus roel-b111ed toucan

G

PAsserforites

Huseleapldae

Bebrom1s rodezicanus Lodrlgues Varbler W

Tchitren hourbanenels nycateber

Eberleldee

Cubernacrix cristate rollow Cardinel or

Ieteridee

xanthopsar flaves seffros-cowlod bleckbird is

Fringl1114e

\section{thofics}

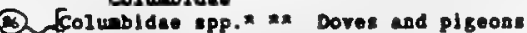

cojub guinea spockled PIgeon

coluba Irldicorques Western Eronze-neped

PIgeon

coluebs livida rock Plgeon

colube unictncea Afop Pigeon

Mesconas ayert Pink Pigeon (A)

Coma capensis Meneque Dove

streptopella deciplens Gournlog collerod Dove

sereptopel1a roseogrised African Collared Dove

serepropel1e sentrorquata Red-eyed Dove

streptopel1a senegalensis Laughiog Dove

streptopel1a turtur Hestern Iurtie Dove

streptope11a vinace Vineceous Dove

Treron calva african Green Plgeon (

Treron walla Bruce's Green Pigeon

rurtur abyssinicus Black-bllled Hood Dove

Turcur afer Blue-spotted Wood Dove

rurtur brehoert Blue-hesded Wood Dove (a)

Trutur typantstria Tabourlne Dove (c)
6

c11

$\mathrm{CH}^{+}$
$\mathrm{CH}+$
$\mathrm{CH}+$
$\mathrm{CH}+$
$\mathrm{CN}$
$\mathrm{CH}+$
$\mathrm{CH}+$
$\mathrm{GH}+$
$\mathrm{CH}+$
$\mathrm{GH}+$
$\mathrm{GH}+$
$\mathrm{GH}+$
$\mathrm{CH}+$
$\mathrm{CH}+$
$\mathrm{GH}+$
$\mathrm{CH}+$
$\mathrm{CH}+$
$\mathrm{GH}+$ serinus letroopygiu mito-runpod casar

serinus rozabicus Iollow-fronted Canary

Iatrizdide (2)

radisa fasclaca cut-throat

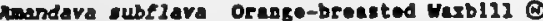

setrilda astrild canon vexblli

satrilda caeruleacese Larender Vaxbl11

Fatr11da melpode orast-cbeoked Waxbli1

sacr1lda erogladyces Black-rumped Haxblil

Lagonosicta larvata meck-faced Firefincb

Lagonosteta rara Black-bellied Fireflack

Lagonosticta rubzicata Brow-backed Firefinch

Laganosticta rufoplcta Ber-brested fireflacb Lagonosticta senegala lod-blilod Fireflocb

lonchura blcolor eleck-breseted anniklo $D$

Lonchura cuculleca Bronze tenalkis ()

Lonchura fringliloides ragplo mennikin $(Q$

lonchura wiabarice silverbli1 (

mandingoa nitidula Green-bucked Iminspot (2)

Mesochar1s caplstrata ulte-cbeeked olivebeck

Migrita bicolor Chestaut-brested Mesro-fincb
E:

$\mathrm{GBT}$

$\mathrm{GB}+$

G.t

C.t.

E.

ats

cos

c.)

E.P.

Cat

E..

cat

Git

$\mathrm{CS}$

ㅌ․

$\mathrm{CH}$

CB.

GB.

Ext 


\section{Appendly III}

Eutrlldides (contlaued)

migrita canicapilla Graj-eromed Megro-finch Migrita fusconota mite-breested Mogro-finch Migrits lutelfrons Pale-fronted Megro-finch ortygosp1zs atricol11s common Quell-finch parmopt1la woodhoures intpocker o

Pholddornis rushiae Iiny Ilt

Pyrenestes ostrinus lack-bellled Soederacker pyeille hypogramica Red-faced pytilis pyt111. phoenicoptera Rod-minged Pytilis spermophaga haesatina Westera Bluebl11 uraegintrus bengalus Red-choeked Cordon-bleu

Ploceldat

Splocelden spp. Menvers

[reblyosplza albifrans Thlck-bllled Weaver Anowlosples 1mberbls Cuckoo Wouver

oubalornis alb1zostris black Buffalo-Wouver (4)

zuplectes afer Yellow-cromed Bishop (0) ouplectes ardens Rod-collarad wldowblrd (4) ruplectes hordesceus Red-cromed Blshop suplectes merourus Yellow-mbouldered

suplectes orts Red Blsbop (i)

malimbus cassini slack-throated ralimbe

Malimbus nalimblcus Crosted Mellabe

Malimbus nttens Blue-billed maliabe

Mallabus rubriceps Rod-bended ralimbe (a)

Malimbus rubricol11s Rod-nocked Mellabe

Milinbu scutatul Red-vented Meliabe

Passer griseus Groj-boedod Sparrow

Petronla dentata Busb Sparrow

plocepassez superc1llosus chestnut-cromed sperrow-Wouver

ploceus albinuche mite-naped Woaver

Ploceus aurantlus orange woever

placeus cucullatus villese verver (1)

Ploceus heuglinl Meuglis" Weaver (1)

placeus lureolus Littlo Wearer (1)

ploceus nelenocepralus sleck-heeded Honver (10

ploceul nigerrimul Bleck Woaver

ploceus nigricoll1: black-necked Wearor

ploceus pelzelnt slender-bliled Weavor

ploceus preussl colden-backed Woaver

plocew superctllosus Conpact Woavor

ploceus tricolor rellow-nantled Woaver

plocents velacus comon Hasked Warer (1)

queles erythrops led-hoeded quelee

sporop1pes frontalls speckle-fronted veover

vidue chalybeate village Indigoblrd (2)

vidun Interjecta Ue11. Paradiso Whydeh

vidua larvacicola Baro Indigoblita

vidur merours Pla-talled ingdab

vidue paradisces comon Paradise unjdah (Q)

vidue rericole Jembendu Indigobled

vidus cogoens1a Togo Paradiso mydah

v1due misanl Hilson' Indigobled

Repti11.

IETTDINAT

Bordideo

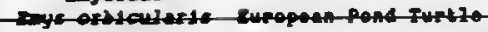

Irtogyebldae

Trianyx trlunguts wil, Soft-shell Iurtle
Appendix III

Prolondusldar

Pelanedusa subrufa Holeoted Turtle

[Pelusios pp. side-necked turtios

pelusiog adansonil hite-brented side-aected

Turtle

Pelus 108 castaneus

Pelus10s gabonensis strlp-backed slde-becked rurt2. (e)

pelusiog niger slack sldo-necked rurtle

$\mathrm{CH}+$

SAURI

Atgeldee

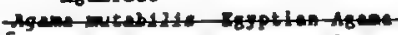

sping-tatiad

cH]

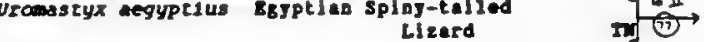

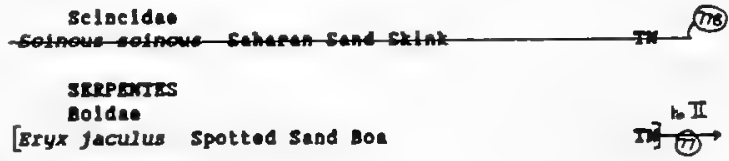

Colubrldae

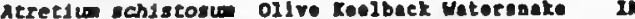

cerberus rhymchops Dog-faced Uater sake II

Ptyas woosus allatle rat sake

Xenochrophis piscator Cuequered Reelbeck

Uaterenare $\odot$

gepldee

waja naja Allatic Cobre I

ophlophague hemeh Rlag cobra II

Tlpordece

vipera russelist Rusnel1" vipar

sophible

cavdra

selenenderide

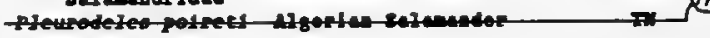

Anve

Auforldee

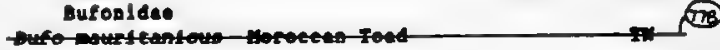

Dy 114 ese

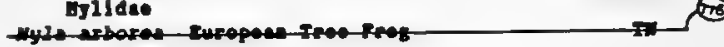

FLO2

Crenpacter

cycas pectinace

Girscre

Cnetu. montanu

newolver:

Talaun hodgsan11

PIPATRICRN

meconops 13 reg1a

m

podocapplase

Podocarpus nerlfollus

MP

Trmactinucus

Q Fetracentran app

ifretracentron inense 
NOTES

\section{APPENDICES I, II \& III}

Aburria jacutinga

Aburria pipile pipile

Anandava subflava

Amazona dufresniana rhodocorytha

Anas clypeata

Anas laysanensis

Andrias spp.

Aonyx congica

Ara glaucogular1s

Arborophila brunneopectus

Aythya nyroca

Balaena spp.

Balaenoptera borealis

Balaenoptera physalus

Boidae spp.

Bolyeria multocarinata

Boocercus eurycerus

Bos gaurus

Bos mutus

Bos sauvel1

Bradypodion spp.

Bradypus variegatus

Bubalornis albirostris

Bubalus depressicornis

Bubalus mindorensis

Bubalus quarlesi

Bubulcus ibis

Cabassous tatouay

Carduelis cucullatus

Carduel1s yarrel111

Casarea dussumteri

Casmerodius albus

Cercopithecus diana

Cervus dama mesopotanicus

Cervus porcinus annamiticus
: Includes generic synonym P1pile

: Includes generic synonym Piplle

: Also referenced es Estrilda subflava or sporaeginthus subflavus

: Formerly listed as Amazona rhodocorytha

: Also referenced as Spatula clypeata

: Also referenced as dnas platyrhynchos laysanensts

: Includes generic synonym Hegalobatrachus

: Also referenced as Aonyx microdon or as Paraonyx microdon

: Often traded under the incorrect designation Ara caninde

: Includes synonym Arborophila oriental1s

- Also referenced as Nyroca nyroca

: Includes generic synonym Eubalaena

: Appendix I populations were the North Pacific stock and the stock in the area from $0^{*}$ longitude to $70^{\circ}$ enst longitude, from the equator to the antarctic continent.

: Appendix II populations were the stock in the North Atlantic off Iceland, the stock in the North Atlantic off Newfoundland and the stock in the area from $40^{\circ}$ south latitude to the Antarctic continent, from $120^{\circ}$ west longitude to $60^{\circ}$ west longitude.

: Includes subfamilies Boinae, Erycinae and Pythoninae

- Formerly listed as Bolyerla spp.

: Also referenced es Tragelaphus eurycerus;

: Includes cynonym Bos frontal1s

: Includes synonym Bos grunniens

Includes generic synonym Novibos

Formerly included in Chamaeleo spp.

: Includes synonyms Bradypus boliviensis and Bradypus griseus

: Includes synonym Bubalornis niger

: Includes generic synonym Anoa

: Includes generic synonym Anoa

: Includes generic synonym Anoa

: Also referenced as ardeola 1 bis

: Includes synonym Cabassous gymurus

: Formerly included in genus Spinus

: Formerly included in genus Splnus

: Formerly listed as Casarea spp.

: Also referenced es Egretta alba

: Includes synonym Cercopithecus roloway

: Includes generic synonym Dama;

includes synonym dama

: Includes generic synonyms $\lambda x t s$ and Hyelaphus 
Cervus porcinus calamianensis

Cervus porc1nus kuhl1

Choramyt1Ius chorus

Civettictis civetta

clella clella

colobus pennanti kirki

colobus rufonttratus

Columba ir1d1torques

Coryphastha minima

Coryphantha sneed11

Crax mitu mitu

CROCODYLIA spp.

Cyclagras gigas

Cygnus columblanus jankowskt1

Dendrocygna bicolor

Dustcyon griseus

Echinomastus erectocentrus

Echinamastus mariposensis

Eploblasma 8pp. (9 entries)

Epioblasma curtisi

\section{Eploblasma florentina}

Bquus hemionus

Erymnochelys madagascarlensis

Bschrichtius robustus

Bstrildidee spp.

Euplectes afer

Buplectes ardens

Euplectes macrourus

Euplectes orix

Eupleres goudot11

Falco peregrinus

Pells caracal

Fel1s rufa esculnapae

Fitz-Roya cupressoides

Galictis vittata

Geochelone elephantopus

Geochelore radiata

ceochelone yniphora

Glaucis dohrnil

Houbarops1s bengalensis
: Includes ceneric synonyms $\lambda \times 1 s$ and Hyelaphus

: Includes generic synonyms $\lambda \times 1 s$ and Hyelaphus

: Formerly included in genus Mytilus

: Includes generic synonym viverra

: Includes synonym Pseudoboa cloella

: Includes synonym Colobus badius kirki

: Includes synonym colobus badius rufomitratus

: Also referenced as Turturoena 1r1ditorques or columba malherbi1 (in part)

: $A 180$ referenced in genus Bscobarta

: Also referenced in genus BScobarla

: Includes generic synonym Mitu

: Includes Alligatoridae, Crocodylidae and Gavialidae

: Also referenced as Hydrodynastes gigas

: Includes synonym Cygnus bewickil jankowsk11

: Includes synonym Dendrocygna fulva

: Includes synonym Dusicyon fulvipes

: Also referenced in genus Neolloydia

: Also referenced in genus Neolloydia

: Also referenced in genus Dysnamia

: Formerly listed as Bploblasma florentina curtisi

: Formerly Iisted as Eploblasma florentina florentina

: Includes synonyms Equus ktang and Equus onager

: Formerly included in Podocnemis spp.

: Includes synonym Eschrichtius glaucus

Previously included in Ploceidae 8 pp.

- Also referenced as Euplectes afra

: Also referenced as Colluspasser ardens

: Also referenced as colluspasser macrourus

: Includes synonym Euplectes franciscanus

: Includes synonym Eupleres major

- Includes synonyms Falco pelegrinoldes and Falco babylonicus

: Also referenced as Lymx caracal; includes generic synonym Caracal

: Also referenced as Lynx rufus esculnapae

: Formerly listed as fitzroya cupressoldes

: Includes synonym Gallctis allamandi

: Also referenced in genus Testudo

: Also referenced in genus Testudo

: Also referenced in genus Testudo

: Formerly included in genus Ramphodon

: Also referenced as Eupodotis bengalensis 


\section{Lagonost1cta larvata}

Leontop1 thecus spp.

Lobe1ra macdougal111

Laschura bicolor

Loschura cucullata

Lonchura frigilloides

Lonchura malabarica

Lutra longicaudis

Lycaste virginalis var. alba

Malimbus rubriceps

Mamillarla pectinifera

Mandingoa nitidula

Manis tetradactyla

Melanochelys tricarinata

\section{MESOGASTROPODA}

Microcycas calocoma

MYTILOIDA

Nasal1s Epp.

Mesoenas meyer1

Nil tava ruecki

Opopsitta diophthalma coxeni

ORCHIDACEAE spp.

\section{ornithoptera spp.}

oryx dammah

otus gurney1

ovis orfentalis ophion

Papio leucophaeus

Papjo sphinx

Papustyla pulcherrima

Parmopt1la woodhouse1

Passer griseus

Peltocephalus dumerillana

Pelusios gabonensis

Physeter macrocephalus

Ploceus cucullatus

Ploceus heuglini

Ploceus Iuteolus

Ploceus melanocephalus

Ploceus velatus

Potanilus capax

PRIMATES spp.

Priodontes maximus

Psammobates geametricus

Pteronetta hartlaubli

Pterygota excelsa

Pygathrix spp.
: Also referenced as Estrilda larvata; includes synonym Lagonosticta vinacea

: Includes generic synonym Leontideus

: Also referenced as Nopalxochla macdougall11

: Includes generic synonym Spermestes

: Includes generic synonym spermestes

: Includes generic Bynonym Spermestes

: Also referenced as Buodice cantans

: Includes synonyms Lutra annectens, Lutra enudris, Lutra incarum and

Lutra platensis

: Also referenced as Lycaste skinner1 var. alba

: Also referenced as Mnaplectes melanotis

- A1so referenced as Solisia pectinata

- A1so referenced as Hypargos nitidulus

: Includes synonym Hanls Ionglcaudata

: Includes generic synonyms Nicoria and Geoemyda (part)

: Formerly listed as Prosobranchia

: Formerly included in CYCADACEAE

: Formerly listed es ANISOMYARIA

: Includes generic synonym Simias

: Also referenced as Columba mayert

: Also referenced as Muscicapa rueck1

: Includes generic synonym Cyclopsitta

: Includes subfamilies Apostasioidere and Cypripedioideae

: Sensu D'Abrera

: Includes synonym Oryx tao

: Also referenced as Mimizuku gurneyi

Includes synonym ovis aries ophion

Includes generic synonym Mandrillus

Includes seneric synonym Mandrillus

Includes generic synonym Papuina

Includes synonym parmopt1la rubrifrons

: Includes synonyms Passer diffusus, Passer gongonensis, Passer suahelicus and Passer swainsonil

: Formerly included in Podocnemis spp.

: Also referenced as Pelusios subniger

Includes synonym Physeter catodon

Includes synonym Ploceus nigriceps

Includes synonym Ploceus atrogular1s

Also referenced as sitagra luteola

- Also referenced as Sitagra

melanocephala

: Includes synonyms Ploceus katangae, Ploceus relchardi, Ploceus ruvet1 and Ploceus vitellinus

: Includes generic synonym Proptera

: Includes family Tupaildae

: Includes synonym Prlodantes giganteus

: Also referenced in senus restudo

: Also referenced as Calrina hartlaub11

: Includes synonym Basilaxylon excelsum

: Includes generic synonym Rhinoplthecus 
Pyrenestes ostrinus

saguinus oedipus

Salacca clemensiana

selenarctos th1betanus

sphlggurus spinosus

stangeria erlopus

Tamandua tetradactyla

Tauraco porphyreolophus

Tetrao mlokosiew1czi

Thamophis couchi hammondi

Toxolasma cylindrella

Tragelaphus speke1

Treron calva

Troganoptera spp.

Troldes $\mathrm{spp}$.

Turtur brehmer1

Turtur tympanistria

UNIONOIDA

Unio nickilniana

Unio tampicoensis tecomatensis

Uraeginthus bengalus

Ursus maritimus

Vidua chalybeata

V1dua paradisaea

villosa trabalis

vulpes zerda

Welwitschia bainesil

Xenochrophis piscator
: Includes synonyms Pyrenestes fromm and Pyrenestes rothsch11d1

: Includes synonym Saguinus geoffroyi

: Formerly listed as zalacca clemensiana

- Also referenced as Ursus thibetanus

: Includes seneric synonym coendou

: Includes synonym Stangeria paradoxa

: Includes synonym Tamandua mexicana

: Formerly included in senus Gallirex

: Includes generic synonym Lyrurus

: Formerly included in Thamophis elegans

: Also referenced in senus Carunculina

: includes generic synonym Taurotragus

: 1180 referenced as Treron austral1s (in part)

: Sensu D'Abrera

: Sensu D'Abrera

: Also referenced as Calopella brehmeri; includes synonym Calopelia puella

: Also referenced as Tympanistria tympanistria

: Formerly listed as MAIADOIDA

: Formerly listed as Unio (Megalonais/?/) nickliniana

: Formerly listed os Unio (Lampsilis/?/) tampicoensis tecomatensis

: Also referenced as Estrilda bengala

: Includes generic synonym Thalarctos

: Also referenced as Hypochera chalybeata; includes synonyms v1dua amauropteryx, vidua centralis, vidua neumanni, Vidua okavangoensis and vidua ultramarina

: Includes synonym Vidua orientalis

: Includes generic synonym Micromya

: Includes generic synonym Fennecus

: Includes synonym Welwitschia mirabilis

: Formerly included in genus Natrix

\section{SPECIAL FLORA NOTES}

Since 1 August 1985 all parts and derivatives of flora listed in Appendix II of the Convention are subject to CITBS controls except:

a) seeds

b) spores and pollen (including pollinia)

c) tissue cultures and flasked seedling cultures

d) fruits and parts and derivatives thereof of naturalized or artificially propagated Cactaceae

e) separate stem joints (pads) and parts and derivatives thereof of naturalized or artificially propagated Opuntia spp. subgenus opuntia

f) separate leaves and parts and derivatives thereof of naturalized or artificially propagated Aloe vera

8) cut flowers of artificially propagated Orchidaceas

h) fruits and parts and derivatives thereof of artificially propagated vanilla spp. 
Prior to 1 August 1985 all parts and derivatives of flora 118 ted in Appendix II of the Convention were subject to CITBS controls except where specifically designated as including only roots, trunks or timber.

This has resulted in the following changes:

Aloe $6 \mathrm{pP}$.

Araucarla araucana

\section{CACTACEAE}

CxatheackaE spp.

CYCADACEAE spp.

DICKSONIACEAs spp.

Dioscorea deltoldea

cualacum sanctum

ORCHIDACEAE SPP.

Panax quinquefolius

Pterygota excelsa

Quercus copeyensis

Rhipsalis spp.

Saussurea lappa

suletenta humilis

ZAMIACBAE spp.

All other species formerly included all parts and derivatives, now includes all parts and derivatives except those in notes a), b), c), f).

formerly referred to timber, now. Includes all parts end derivatives except those in notes a), b), c).

formerly included all parts and derivatives, now includes all parts and derivatives except those in notes a), b), c), d), e).

formerly included just trunks, now includes all parts and derivatives except those in notes a), b), c).

formerly included all parts and derivatives, now includes all parts and derivatives except those in notes b), c).

formerly included just trunks, now includes all parts and derivatives except those in notes a), b), c).

formerly included just roots, now includes all parts and derivatives except those in notes a), b), c).

formerly included just timber, now includes all parts and derivatives except those in notes a), b), c).

formerly included all parts and derivatives, now includes all parts and derivatives except those in notes a), b), c), b), b).

formerly included just roots, now includes just roots and readily recognizable parts thereof.

formerly included just timber, now includes all parts and derivatives except those in notes a), b), c).

formerly included just timber, now includes all parts and derivatives except those in notes a), b), c).

formerly included all parts and derivatives, now includes all parts and derivatives except those in notes a), b), c), d).

Formerly included just roots, but now listed in Appendix $I$.

formerly included just $t$ imber, now includes all parts and derivatives except those in notes a), b), c).

formerly included all parts and derivatives, now includes all parts and derivatives except those in notes b), c).

includes all parts and derivatives except those in notes a), b), c). 
i

. 
PART 2

\section{A RECORD OF SPECIFIC RESERVATIONS EHTERED BY PARTIES TO CITES}

The scientific names in these lists are based on the official Appendices $I$ and II 18 at 29 August 1986 and Appendix III as at 5 March 1986. and on previous editions of the official Appendices, produced by the CITES secretariat. The information included has also been obtained from documents produced by the cIrEs secreteriat.

Wildlife Trade Monitoring Unit

Copyright (C) 1986. IUCN Conservation Monitoring Centre. $219 \mathrm{c}$ Huntingdon Road, Cambridge CB3 ODL, UK. 
. 


\section{Explanation}

The list on the following pages details all reservations that have been entered by Parties to the Convention on

International Irade in Endangered Species of Wild Fauna and Flora. The dates given are the date of entry into force of each reservation and, where relevant, the date of withdrawal of the reservation. As in the CITES text, "species" means any species, subspecies or geographically separate population thereof.

The numbered annotations refer to the notes on pages 14 and 15 and a list of the I.S.O. country codes used is included on page 16.

Key

r - denotes a reservation withdrawn as a result of the removal of a species from the Appendices

8 - denotes a reservation succeeded by a later reservation involving higher taxonomic group

$t$ - denotes a reservation withdrawn as a result of a species having been transferred from Appendix I to Appendix II

I - denotes a reservation withdrawn as a result of a species having been transferred from Appendix II to Appendix I or from Appendix III to Appendix II

! - indicates reservations entered by Austria with respect to all amendments to Appendices I and II adopted at the fourth and fifth meetings of the Conference of the Parties held in Gaborone. Botswana in April 1983 and Buenos Aires, Argentina in April 1985 
Appendix I

raune

Counter sntered ulthdrawn

\section{enellis}

\section{remurts}

cobldev

Lagoengla plavicauda

AT1 $29107 / 83$

\section{Coreopl tbeclate}

pygathrix epp. 1

craces

2lpblsdes

serard1ue epp

Berardiu blidil

myperoodon ePP.

\section{Phyeterldee}

Physecer acrocephalus 2

Delpblaidee

socal14 APP.

sousa epp.

Pocoes 1dee

meophocsens phoceenoldes

\section{enchebtere}

Eschrlchelus rabustus 3

ca

ATf 29/07/83

SU $29107 / 83$

IP 29/07/83

AI! $29 / 07 / 83$

so $29107 / 83$

JP $06 / 06 / 81$

wo $06 / 06 / 81$

CA $28 / 06 / 79 \quad 29 / 10 / 82$

zA $28 / 06 / 79 \quad 17 / 02 / 81$

CA $28 / 06 / 79 \quad 29 / 10 / 82$

IR $28 / 06 / 79 \quad 17 / 02 / 81$

Ca 28/06/79 29/10/82

Beleenopterldee

belaenoptera ecutorostata

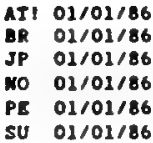

balsenopter borealls

population: notes JP 06/06/B

MO $06 / 06 / 81$

population: see note 6 AU $04 / 02 / 77$ 27/08/8

ch $04 / 02 / 77 \quad 29 / 10 / 82$

sU 04102177

in $04102 / 71$ 17/02/81

baj senopiers edens

\section{Append II II}

Couater Entered Ulthdraw
CA $28 / 06 / 79 \quad 30 / 20 / 80$

24 $28 / 06 / 79 \quad 17 / 02 / 81$ 
Appendis I

Country Entered Hithdrewn

\begin{abstract}
Belenopterlace (continued)
\end{abstract}

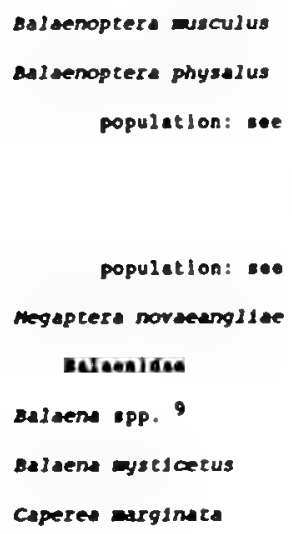

\section{Beloenifica}

Uraldee

Uzsus awericanus emons11

ursus arctos 1sabellinus

CA $09 / 07 / 75$ 04/02/775

Hertelldae

wera lutra

posldas

Pells caracel 11

pells conoolor cougar

rells rubiginces 12

prososcroka

Eepbestidae

rlepros maxims

$$
\begin{aligned}
& \text { ca } 09 / 07 / 75 \text { 21/08/78 } \\
& \text { CA } 09 / 07 / 75 \text { 11/08/78 } \\
& \text { AI! 01/02186 } \\
& \text { AR } 01 / 01 / 86 \\
& \text { PE } 01 / 01 / 86
\end{aligned}
$$

ad aenoptera physalus?

AppopdI II

Counter entered ulthdram
CH $28 / 06 / 79$

\section{Can1s. Iupus 26 \\ cants lupue crassodon \\ Can1s Iupus 1rremotus \\ vulpes zerda 27}

Ursus arctos 28

ursus arctos 29

ursus merse1ms

s) $04 / 02177$ martes anertcand atraca

Fel1: bengalenst:

$$
\begin{aligned}
& \text { AIl 01/08/85 } \\
& \text { bengalens1. } 30 \\
& \text { CA } 09 / 07 / 73 \text { 25/04/77 } \\
& \text { sU } 04 / 02 / 77
\end{aligned}
$$

pells concolor atsoulensis rells lym

Ca $09107 / 7504102 / 177^{2}$

\author{
su $04 / 02 / 77$ \\ ca $09 / 07 / 75$ 25/04/77 \\ ca 09/07/73 13/04/77 \\ AII 03/08/8s
}

ce 09/07/75 29/10/82

AT1 29/07/83

ca 09/07/7s 15/04/77

Ge (for MR)

$04 / 02 / 77 \quad 03 / 07 / 78$ 
Append $1 \mathrm{xI}$

Countey Entered withdrawn

PeEssonactras

Equidac

\section{squus africanus \\ Arfoactra \\ cervldee}

nostiu app. 13

Noschus soschiferus it

mentecus crinifrons

\section{novides}

iddax nascaculacus

Blson bisan athabascae

Oryx denmah

Pantrolops hodgsont

Aves

ETxuTाOnI rontes

stratbloaldae

struthlo canelus 15

rimpenitrois

Pelocaldet

pelecanus crispus

crocartroweds

cleosildes

Jabiru aycter1a

Proevi eopterlace

\section{Arsartones}

Anatidea

Brante canadensis

levoopareia

rascourrosers

Aeelpitridae

Faleondae

AIt $29107 / 83$
CA $09 / 07 / 73 \quad 29 / 10 / 82$

AT! $29 / 07 / 83$

JP $04 / 11 / 80$

DR $16 / 02 / 79$

$01 / 01 / 80$

AT! 01/08/85

AII $29107 / 83$

Aร! $29107 / 83$

CH $28 / 06 / 79$

AIt 29/07/83

ATI 29/07/83

ATI $29 / 07 / 83$

ATt $01 / 08 / 83$
ATt $01108 / 85$

CA $09107 / 75$ is/04/77
Appendis II

Country Entered Nitbdram noschus epp.

moschus epp. 31

moschus aschiferus 32

Amotzagus lervia

audorcas taxicolor

cephalophus dorsalls

cephalophus jencink1

cephalophus oglibyl

Cephalophus sylvicultot

cephalophus rebra

ov1s caredens $1 \mathrm{~s}$

Phoenlcopterldee spp. 33

Anser albifrons gabell1.

oxyura leucocephala

Aquila chrysaetos

Falconldee epp. 34

ca 09/07/7s 15/04/77
DR $16 / 02 / 7901 / 01 / 80$

ATt $29 / 07 / 83$

SP $\quad 29 / 07 / 83$

AT! $29 / 07 / 83$

ATI 01/08/8s

AI $29 / 07 / 83$

AI $29 / 07 / 83$

AT) $29107 / 83$

AT! $29 / 07 / 83$

AT! $29107 / 83$

Ca $09 / 07 / 75 \quad 29 / 10 / 87$ 
Appendix I

Country Entered Withdrawn

GQUTFOHES

Iurodcidee

Pedlonoldee

cruldae

otidides

Chlanydorls undulata

GKALEDTIPORER

Scolopacidae

Nuenlus tenulrostris

COUTBIPOREs

Colablden

Cajoenas nicobarlca

siructroms

Pelttacidee
CH $28 / 06 / 79$

AI) $29 / 07 / 83$

CH $28 / 06 / 79$
AppendiI II

Country Entered Withdram

rurnix melanogaster

CH $28 / 06 / 79$

Pedlonomus torquatus

CH $28 / 06 / 79$

Gruldee epp. 35

At! $01 / 08 / 85$

Anthropoldes virgo

AT! $29 / 07 / 83$

$\begin{array}{lll}\text { Cyanol1seus pacagonus byrons } & \text { CH } 28 / 06 / 79 \\ \text { PSITIACIFORMS SPP. } 36 & \text { CH } & 06 / 06 / 81 \\ & \text { LI } 06 / 06 / 81\end{array}$

Ara abigua

Ara glaucogular1s 16

Ara macao

ara rubrogenys

Ognorhymchus 1cterots
AI! 02/08/85

AT! $29 / 07 / 83$

AT! $01 / 08 / 85$

CH $01 / 08 / 85$

LI $01 / 08 / 85$

SR $01 / 08 / 85$

AT! $29 / 07 / 83$

AT! $29 / 07 / 83$

reservation not eppliceble to:

Anazona agll1s

mazona collarta

Mazona dufresnland "h.

Anodorhymchus hyacinthinus

Ara spp.

cacatua cenulroser1s

Calyptorhymchus lathand

Coracopsis sp.

Cyanorampus alherb1

Cyanoraphus unfcolor

Bunymphicus cornutus

Neophena splendida

agnorhymchus lcrezatis

polcephalus robustus

Polytelis alexandrae

Probosciger aterrinus

Prosopela Ipp.

Psephotus haematogaster nare thae

psictrichas fulg1dus

Tanygnathus luclanensis

Trichoglosilnte app.

reservation not applicable to:

Cyanollseus patagonus byront

Switzerland and Llechtenstein aloo held reservatlons with regard to: tbe apecles anked axcept coracopis

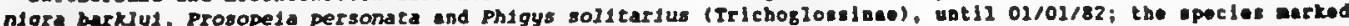

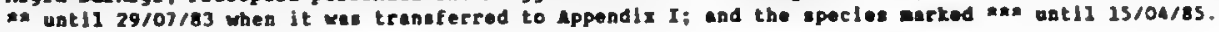


Appendix I

Countri Encered Witharawn

eepti11.

all llated opeclos

TETrornata

crelonildes

crelonia sydas 17

Erecnochelys 19bricata

Lepldochelys ollvace

normockelifide*

termochelys coriaces

chocoprus

Allsetorldee

calman lat1rostris

Melanosuchus niger

Crocodj214e

crocodylue scutus 18

Crocodylus cacapheactus

crocodylus ntioticus 19

Crocodylu porosus 20

Crocodylus stansis

osteolenus cecraspls

gure

Varesides

varanus bengalensis

varanus tavecaens

varanus ardecus

27 $17 / 08 / 81$
CA (FOF HR) $31 / 20 / 76 \quad 03 / 07 / 78$

a) 11eted opeclos

$\begin{array}{lll}\text { IR } & 09 / 08 / 78 & 10 / 12 / 84 \\ \text { IT } & 31 / 12 / 79 & 01 / 01 / 84\end{array}$

JP $04 / 11 / 80$

SR 13/02/81

FR $09 / 08 / 78 \quad 10 / 12 / 84$

JP $04 / 11 / 80$

JP $04 / 21 / 80$

SR $15 / 02 / 81$

II $31 / 12 / 7901 / 01 / 84$

PR $09 / 08 / 78 \quad 10 / 12 / 84$

CH $28 / 06 / 7906 / 06 / 81$

AI 27/04/82

FR 09/08/78 10/12/84

II $31 / 12 / 7901 / 01 / 84$

24 $22 / 02 / 81$

av 12102118

IR $09 / 08 / 78 \quad 10 / 12 / 8$

II $31 / 22 / 7901 / 01 / 8$

SD $24 / 01 / 83$

24 22/02/81

AI 27/04/82

CH 28/06/79 01/01/83

OR $28 / 06 / 7902 / 07 / 82$

PR 28/06/79 10/12/84

II $31 / 22 / 7901 / 01 / 84$

JP $04 / 11 / 80$

TH $21 / 04 / 83$

TH $21 / 04 / 83$

Fh $09 / 08 / 78 \quad 10 / 12 / 84$

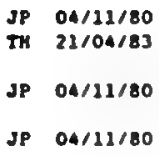

AppendIr II

Country Intered yltbdraw

Ca (for HR)

$31 / 10 / 76 \quad 03 / 07 / 78$

Crocodylus niloclcue 37

ATI $01 / 08 / 85$

crocodylus poroeut 38

at $01 / 08 / 85$

IH $21 / 04 / 83$ 
Appendis :

Country Entered withdratn
Appendix II

Countri Entered Hitheram
Itiprotes

soldet

splcraces monens 15

eleplde*

impibla

Allus:

Hobetrachldes

seridee

\section{Heen}

Actrosmiroms

aclponerldea

Acipenser brevirostru

Actpenser aryrhymchus

Acipenser stur10

sefwom romes

reloontace

coregamu ispenve

ctrerriroves

CTpeleldee

\section{perciromas}

Pereldee

stirostedion vicreu

glaucen
AT! $29 / 07 / 83$

Pytion molurus biviteatus Python reticuletus

Hoplocephslus bungaroldes

AT $01 / 08 / 85$

Rheobatrachus epp.

AT! 01/08/8s

Reme hexedectyle

Ca $09 / 07 / 75$ 13/04/77

CA $09 / 07 / 75 \quad 28 / 06 / 79^{t}$

AT) $29 / 07 / 83$

CA $09 / 07 / 75 \quad 29 / 10 / 82$

CA $09 / 07 / 75 \quad 29 / 10 / 82$
Rand tgerine

Aclpenser fulvescens

CA 09/07/75 29/10/82

AI! 01/08/8s

cH $01 / 08 / 85$

(L) $01 / 08 / 85$

aT) 01/08/85

ct $01 / 08 / 85$

LI $01 / 08 / 83$
C1 $06 / 06 / 81$

LI $06 / 06 / 81$

Serects

Lepropptra

Peplllooldee

Orn1 thopter. ipp. 39

rrogonoptera EP. 39

rrotdes ap.
DR $16 / 02 / 79 \quad 01 / 02 / 80$

DR $16 / 02 / 79 \quad 01 / 01 / 80$

DR $16 / 02 / 7901 / 01 / 80$

\section{Areesalde}

morer 
Appendis I

Countey Bntered Wlehdrawn
Appendla II

CountrI Entered Withdram

Choromyt1lus chorus 40

Сम $28 / 06 / 79$

VENEROIDA

Iridacnidee

Tr1dacridae sp

Tridacna derasa

rridacna glgas

AT! $01 / 08 / 85$

AT! $29 / 07 / 83$

AT! $29 / 07 / 83$

Ser1acopora epp.

poclllopora pp.

seylophora epp.

Aeroporldee

Acropoza EPP.

Pavona epp.

Fungla spp.

Halomitra epp.

Polyphyll1d epp.

Ferlldee

Eerullnldae

ques dae

Pectiolldee

Cerjophy111ddao

Favia spp.

Platygyra sp.

Merulina $\mathrm{PP}$.

Lobophy111a spp.

Pect1n1a spp.

Euphylz1a epp.

M1llepora ipp.

Hellopoza sp.

rublpota PP.
At: $01 / 08 / 85$

AT $01 / 08 / 85$

AI! $01 / 08 / 85$

AI1 $01 / 08 / 83$

AI $01 / 08 / 85$

AI! 01/08/83

AI! $01 / 08 / 85$

AI! $01 / 08 / 85$

AT! 01/08/85

AT! $01 / 08 / 85$

AT! $01 / 08 / 85$

AI $01 / 08 / 85$

AI $01 / 08 / 85$

AI! $01 / 08 / 85$

AT $01 / 08 / 85$

ATt $01108 / 85$
AT! $01 / 08 / 85$ 
Appendiz I

Country entered Withdrewn

TLOR

\section{Agavacese}

Agave axtzonica

Mgave parviflora

molina Interrata

APOCTUCER

Pachypodicio nanaquanum

\section{mavenRtacear}

Azaucarla araucana 21

MCLap udacen:

Broubaces

cericens

Anets srocactus tobusch11

arlocarpus agavioldes

Artocarpus scapharostrus

Ariocarpus exigonus

Aztekiw ritrer:

sackeberg1a wittaris

coryphanthd atnima 22

coryphantha sneed 1122

coryphantho werdermannil

Echinocereus IIndsayl

Beh1nonastus erectocentrus ${ }^{23}$

sch1nomatus

martposens $1 \mathrm{~s}^{23}$

zevehtenbergta principls

Lobetra macdougal11124

Mandizlar1a pectinifera 25

mamsllaria plumosa
AI! 29/07/83

AT! $29 / 07 / 83$

AI! $29 / 07 / 83$

CH $06 / 06 / 8101 / 01 / 83$ LI $06 / 06 / 81 \quad 01 / 01 / 83$

CH 28/06/79 01/01/83 FLorA sp. 41 AT! $01 / 08 / 85$

Agave v1ctoriae-reginae

AI! $29 / 07 / 83$

CH $28 / 06 / 79 \quad 01 / 01 / 83$

Ceropeg1a sp.

Frered indica

CH $28 / 06 / 7901 / 01 / 83$

Byblys epp.

CH 28/06/79 01/01/83

CACTACEAE app. 42

a $09 / 07173$ 25/04/77

CACTACRAE ipp. 43

aT $29108 / 86$ 


\section{Appendix I}

Country Entered Hithorawn

CACTACEs

(contloued)

mamillarta sollstotdes

Obregonta denegr11

Pediocactus bradyl

Pedlocactus despalnt1

Pedlacactus bnowltonld

Pedlocactus papyracanthus

Pedtocactus paradinel

Pedlacactus peeblesianus

Pedlocactus stlert

Pedlocactus winkleri

Pelecyphora aselliforms

Pelecyphor strob1liforms s

Sclerocactus glaucus

sclerocactus nesae-verdae

selerocactus pubispinus

sclerocactus mightdae

Turblnicarpus spp.

Nilcoxia schmosil1

cepraveracers

croonmancer

coepositar

Saussured lappa

\section{cerssulachas}

Dudleya stolonifera

Dudleyd crasklae

DIPExsterex

\section{crucacer}

Fouguteriacens

Fougulerda fasciculata

Fouquier1a purpus11

AI! 29/07/83
AT! $29 / 07 / 83$

CH $06 / 06 / 81 \quad 01 / 01 / 83$

LI $06 / 06 / 8101 / 01 / 83$

AT! $29 / 07 / 83$

AIf $29 / 07 / 83$

AT! $29 / 07 / 83$

AT! $29 / 07 / 83$

AI! $29 / 07 / 83$

At: $29 / 07 / 83$

AI $29 / 07 / 83$

AT! $29 / 07 / 83$

CH $06 / 06 / 81 \quad 01 / 01 / 83$

LI $06 / 06 / 8101 / 01 / 83$

CH $06 / 06 / 81 \quad 01 / 01 / 83$

LI $06 / 06 / 8101 / 01 / 83$

AT! $29 / 07 / 83$

AT! $29 / 07 / 83$

AI! $29 / 07 / 83$

AT! $29 / 07 / 83$

AT1 29/07/83

Cephalotus foll1cular1s

CHLOATtHACRAs epp. 4 ,

AI! $01 / 08 / 83$

AT: $29 / 07 / 83$

AI! $29 / 07 / 83$

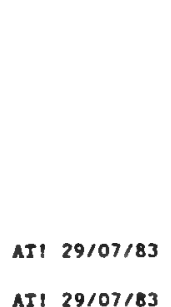

\section{Appendil II}

Country Entered Witbdram 
Appendis I

Country Entered Hithdrawn

HWODDRECER

mrtacear

meportracens

orchroacen:

\section{Renanthera inschootiana}

Vanda coerulea

CH $28 / 06 / 79$

CH $28 / 06 / 79$
CH $06 / 06 / 81 \quad 02 / 01 / 83$

LI $06 / 06 / 8101 / 01 / 83$
Nepenthes rajah

Nigozanthos ipP.

Macropldia fullginosa

verticord1a spp.

ORCHIDACEAE IPP.

(all those not in APp. I)

Lewisia cotyledon

Lewis1a naguire1

Lew1sta serrata

Lewisia areedy1

protzaczer

SArEACEACACER

Sarracenla alabanens $1 \mathrm{~s}$

alabamensis CH 06/06/81 01/02/83

LI $06 / 06 / 81 \quad 01 / 01 / 83$

Sarracen1* jones 11

sarracenta oreoph1la
CH $06 / 06 / 8101 / 01 / 83$

LI $06 / 06 / 8101 / 01 / 83$

LI $06 / 06 / 81 \quad 01 / 01 / 83$
CH $06 / 06 / 8101 / 01 / 83$

Tatacer

THETSRACEAR

zurraces

ceracozanta sp.

Append IX II

Country Entered Hithdram

\author{
CH $28 / 06 / 7901 / 01 / 83$ \\ CH $28 / 06 / 79 \quad 01 / 01 / 83$ \\ CH 28/06/79 $01 / 01 / 83$
}

CA $09 / 07 / 75 \quad 15 / 04 / 77$
Danks1a spp.

Conospermum sp.

Dryandra polycephala

xylonelum ipp.

Boronla sp.

crowed app.

celeznowla verrucosa

Darlingtonia callfornica

Canell1a chrysartha

P1melea physodes
AT! 01/08/85

CH $28 / 06 / 7901 / 02 / 83$
AT! $29 / 07 / 83$

AT! $29107 / 83$

AT $29 / 07 / 83$

CH $28 / 06 / 79 \quad 01 / 01 / 83$

CH $28 / 06 / 7901 / 01 / 83$

CH 28/06/79 $01 / 01 / 83$

CH 28/06/79 01/01/83

CH $28 / 06 / 7901 / 01 / 83$

CH 28/06/79 01/01/83

CH $28 / 06 / 7901 / 01 / 83$

CH $06 / 06 / 8101 / 01 / 83$

LI $06 / 06 / 8101 / 01 / 83$

- These speles were deleted from the Appendlces efter the fourth aeting of the Conference of the Parties held in Gaborone. Botzwane, In Apr II 1983. 
AppendII III

reve

Counter Entered Mlthdrawn

Memedle

CKIDPTEL

Pbjilostonidar

vapyrops inneatus (UX)

Dr 24/10/77 01/01/84

\section{morata}

eredypodidee

oredypue grimeus (CR) is Choloepus hoffand (CR)

\section{Desypodideo}

CAbessous central1s (CR) cabassous tatouay (UY)

Phocioors

valdae

\section{Mand glganter (CH) wands cecradactyla (CH) nands ericuspl: (GH)}

\section{nodorra}

seluridas

Ip1xeru: eb11 (CH)

scluru deppel (CR)

\section{Docalurides}

Ancalurus opP. (GH)

Idlurus sPP. (CH)

Gatrledine

Hystr1x opp. (CH)

rethleostides

spsiggurus spinosus (UY)

crsacen

Pretesietide

Pontopor1a blanville1 (UY)

monodontidee

manodan manocros (CA)

\section{cistroe}

cendere

rulpes zerds (IN)

\section{Procjogldae}

Dassaricyon gabb11 (CR) pasarjecus sludchrased (CR) Masua nasua solicarla (UT)

tustellase

Gallctis viteta (CR) mellivora capened: (GH")
DR 24/10/77 01/01/84 DR 24/10/77 01/01/84 or 24/10/77 01/01/84

DF 24/10/77 01/02/84

DR 24/10/77 01/01/84

DE 24/10/77 01/01/84

OR $24 / 10 / 77 \quad 01 / 01 / 84$

DR 24/10/77 01/01/84

DR 24/10/77 01/01/84

DK 24/10/77 01/02/84

or 24/10/78 01/02/84

DR $24 / 10 / 77 \quad 01 / 01 / 84$

or $24 / 10 / 7728 / 06 / 79^{T}$

Dr $24 / 10 / 77 \quad 28 / 08 / 79^{T}$

Dr 24/10/77 01/01/84

Lar

Hagedash1e hagedesh (G

Threskiornts sechsoptcu (CH)

\section{Aisers rotes:}

Anstldee

Anetidee epp. (CH) 47

racooniforites

icelpltridee

DR 24/10/77 01/01/84

DR 24/10/77 01/01/84
Lapribis rera (GK)

\section{Append I III}

Couater Eotered withdrem

DR 24/10/77 01/01/84

OR $24 / 10 / 77 \quad 01 / 01 / 84$

or 24/10/77 01/01/84

Dr 24/10/77 02/01/84

DR $24 / 10 / 77 \quad 29 / 07 / 83^{2}$

DF $24 / 10 / 7701 / 01 / 84$

DF $24 / 10 / 77 \quad 02 / 01 / 84$

Dx $24 / 10 / 77$ 01/01/84

of $24 / 10 / 7701 / 01 / 84$

DR 24/10/77 01/01/84

of $24 / 20 / 7701 / 02 / 84$

DR 24/10/77 01/02/84

Dr $24 / 10 / 77$ os/06/81

DE 24/10/77 01/02/84

DR $24 / 10 / 77$ 01/02/84

Dr 24/10/77 01/03/84

DF 24/20/77 01/01/84

DF $24 / 10 / 77 \quad 01 / 01 / 84$

DR 24/20/77 01/02/84

DF 24/10/77 01/01/84

Acelpltrides epp. (CR) 4 a $24 / 10 / 77$ 01/01/84

- Also Ilated in Appendiz III fron $24 / 04 / 78$ by Botawene 


\section{Appendis 111}

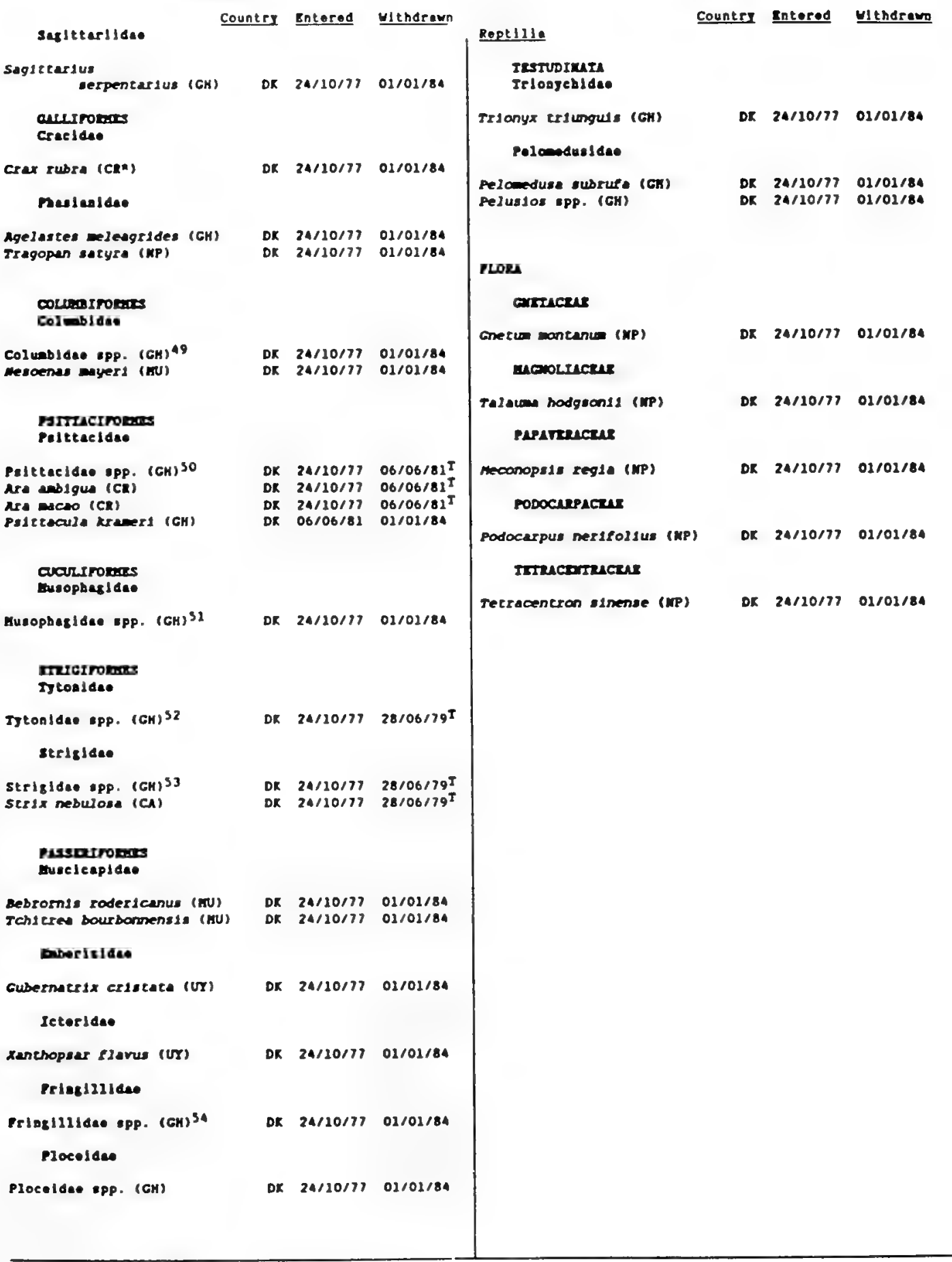

\section{Appendix III}

Countre Entered Ulthdrevo 


\section{Appendix 1}

1. Iaeludes senerle ejnonga Rrinoplesecus

2. Ineludes iynosy physeter catodon

3. Taelude: syonge ischrlahtlus gleucus

4. Krept population of Mest Greenland

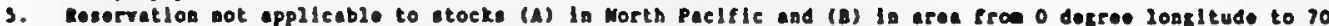
degrees east longltude roe the equator to the Astaretse continent

6. Stocke (A) Ia Morth Pactelic and (B) lo area fron O degree Iongltude to 70 degrees eagt longltude froo the equator to the Anterctic continent

7. Renervetion not applicebl to etocks (A) In Morth atientse oft Ieolend, (B) in Wortb atjeatle off

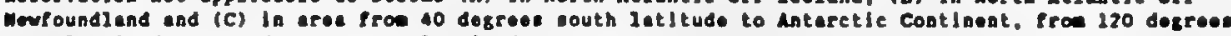
wit longltude to 60 degrees west longltude

B. Stocks (A) In Morth Atlantle ofl Iceland, (B) In Morth Atlentle off Mowfoundiand and (C) In aree froe 40 degrees souts letitude to Anterctie Continent, fron 120 degrees west losiltude to 60 degrees wat lonstivde

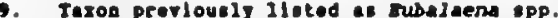

10. Populations of Bbuten, Indla, Mopel end Pakisten

11. Asias population (also liated al Lym ceracel, Ineludes the generle egnonge caracal)

12. Indien population

13. Populatione of Afghenleten, Bhutan, surne, Indie, Mopel and Pakleten

14. Mikeleyas population

13. Populations of Algerle, Burkine, Camroon, the Centrel African Ropublic, Cbed, Mell, Meuritanle, Morocco, Miger, Migerle, Senegel and Suden

16. Often treded under the incorrect deilgnatlon pra cansinde

17. Reservation not eppliceble to the Australlen population

18. Population of the Unlted Stater of Merice

19. Reept population of 2lmbebre end populetione of the following countrles: Ceaeroon, Congo, Kenje, Medesatcer, melavl, Mozenblque, Suden. Unlted Republle of tanzenle and zenble

20. Exept population of Papue Meu Gulnec and, Ince 01/08/85, the population of Indonerle and the ranebed population of australle

21. Population of chile

22. Aleo referenced in genus Escoberte

23. Also referenced in genus Meolloydse

24. Also reforenced al Mopelxochla atcdouga1111

25. Also referenced at Sollsila pectinate

\section{Appendix II}

26. Ireept populations of thutan, Indla, Wepal and Paklatan

27. Ineluder generle iynonym Fennecus

28. All Morth deorlcen oubepecler

29. Population of Buropt, except USSR

30. Chlaese populatlos

31. Exept populetiose of Afghenleten, shutan, surnh, Indle, Mopil end paklsteo

32. Except himalejan populatlon

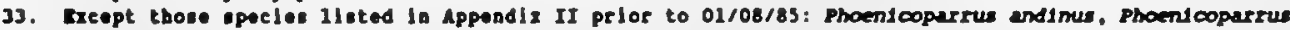
jasess, thoentcopterus chllensis and phoenicopterus ruber

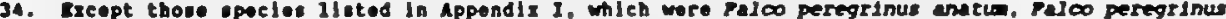

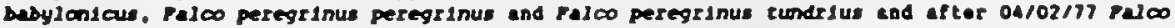
arene. Faloo newtoni aldabrenus. Falo pereginus end ralco punctatü

33. Excopt those spoclor llated in Appondiz I: Grus americana, Grus candensis mesloces, Grus canedens1s pulls, Grus japonensis. Grus levoogeranus, Grus manecha, Grus nigricolls: and Grue rip10 and thome lleted in Appendix II prior to 01/08/8s: anthropoldes virgo, salearice reguloru. and Grus canedensis precensis.

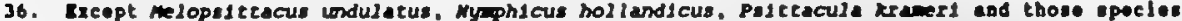

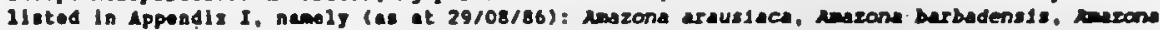

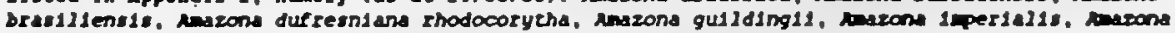

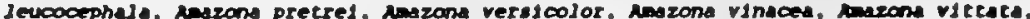

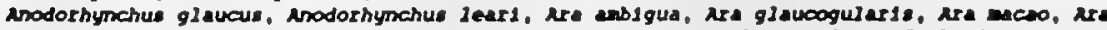
rubrogenys, Areeinge guaroube, Cyanopsicta sp1x11, Cydaroraphus auriceps forbest, Cyenoraphus

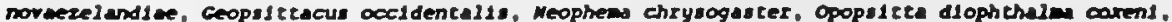

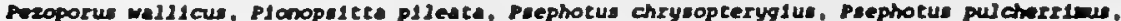

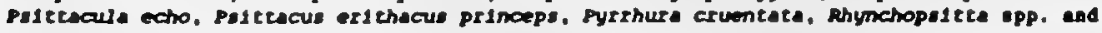
strigops sebropt11us

37. Populatioge of the folloulng countrles: Cameroon, Congo, konje, Madegencer, Melewl, Mozablque, suden, Ualtod Ropubile of Ianzanle and zable

38. Populatios of Indoberle and tbe reached population of Australle

39. Sengu D'Abrere

40. Pormenly lleted as meslus chorue 


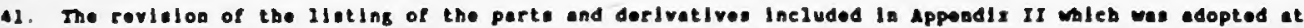

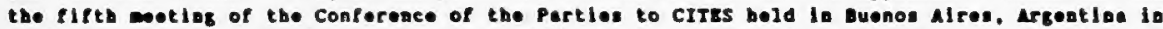
April/Kay 198s resulted in the Pollowling additions to Appendix II, with respect to inleb Austrie has entered reservatlose:

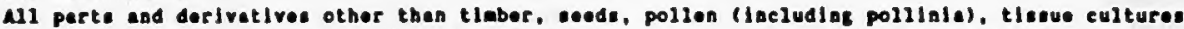

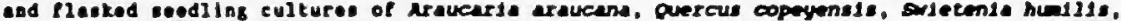
pterygote excelsa and Gunacu sanctu.

All parts ad derlvatives other then roots. eeeds, polles (Ineluding polilale), tisaue cultures and rlenked seodilng cultures of Dloscoree deltoldes

All parte and derivatives other than trunks, eeds, polien (Ineluding pollinia), tiesue cultures

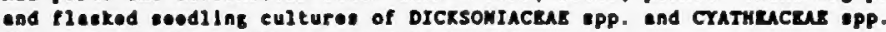

The seods of CTCADACRAs epp. and zariacens spp.

42. A11 upecles of the fasily in the Nertees

43. Uatil 29 August 1986 , the lleting of Cactecea opp. In Appendix II wee ennoteted all epecles of the

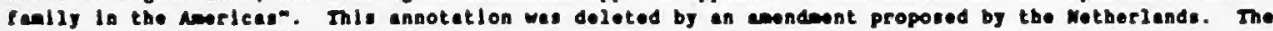
proposel teted that there wre four possble Interpretetlons of the anaoteted 11 etlog of cactacese ipp. In Apposdi II:

- All cectl regerdlos of thele origin and statue leltbor wild-collected or artiflelally propagated) were covered by CITEs; or

b. All cactl taken lo the wild In the Amerleas or artiflelally propagated in the aneleas we corered by the Convention. Therefore, cact artifleleliy propegated or froe acturallzed

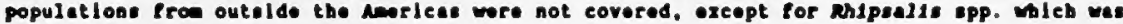
llated eeparately; or

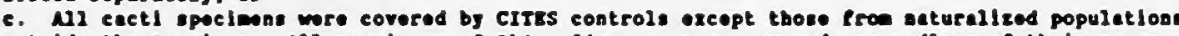

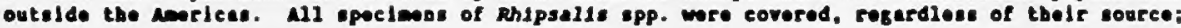
or

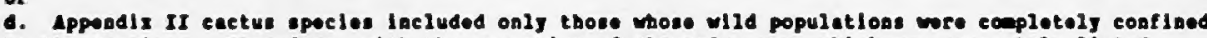

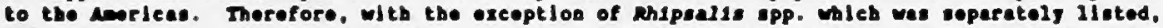

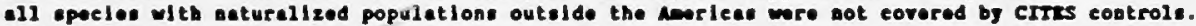
Oe the besis of these laterpretetione of the annotetion, lta deiotion resulted in one of the followias four aditios to Appendix II with respect to mich auterle has ontered a reservatlon:

A. Mose: or

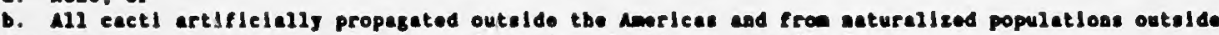

the Anoflcas, except Rhipsalis epp. and opecles already in Appondix I; or

C. All aturalized populatione of appendiz II cact outside tbe anerleat axeopt tbose of

Phipsal1: epp ; or

d. All species of cactus thet heve neturelized populetlons outside the anclese oxcept

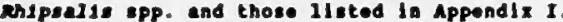

44. Australien populations

\section{Appendis III}

45. Incorporeted tato sradypus veriegacus, which was lieted in Appendix II

46. Except Rhe terlean albescens (Included In Appendla II)

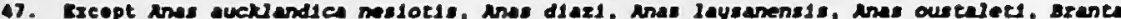

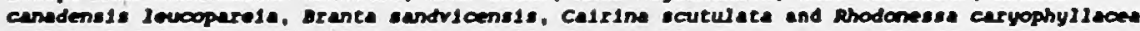

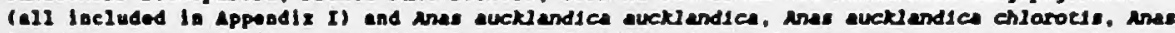

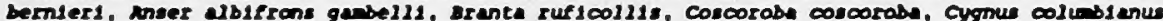

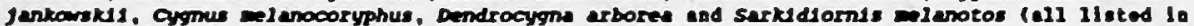
Append I I II)

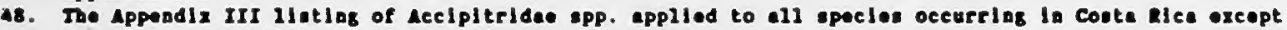
Marpla harpyje whleb we elready Ilated In Appendlx I

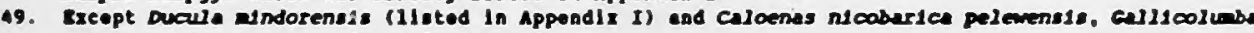
lurentea, coura epp. (Ileted It Appendiz II)

30. Except those specles Ileted in Appendlx or II (4 Februery 1977 )

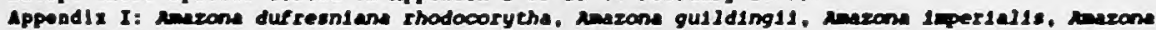

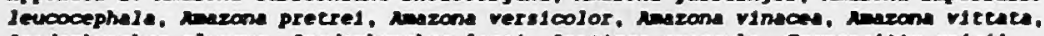

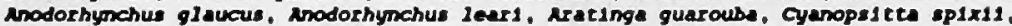
cyanoraphus urlceps forbes1, Cyanoraphus novaezeland1ae, Ceopeltracus occidentalls, Meopheas chrysogaster. Peroporu wellicus. Plonopaltea plieata. Psephotus chrysopteryglus. Prephotus

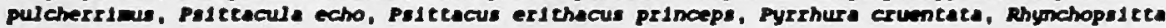
pachyrhymat and strigops hebroptilus

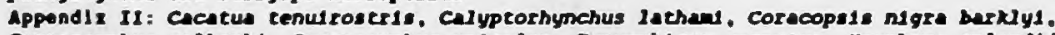
Cyanoraphus alherbl, cyanoraphus unicolor, suny phicus cornurus, weophere eplendida, opopstre diophthale caren1. Polcephelus robustus, Polytells alexandree, Probosclger

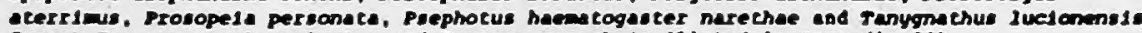

31. Except reuraco porphyreolophus and rauraco coryehald (1lated in Appendis II)

32. Except Tyeo soungeres (IIsted in Appendix I)

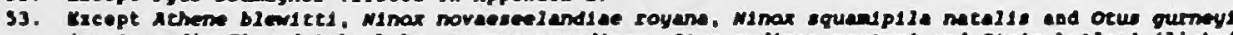
(on Appendix I) and subo bubo, wycted scandiacs, otus nudipes newtoni end strix butleri (IIsted In Appandix II)

54. Except Carduel1s cucullatus (AppendIx I) and Carduells yartell11 (Appendix II) 
Llet of I.S.O. country codes used

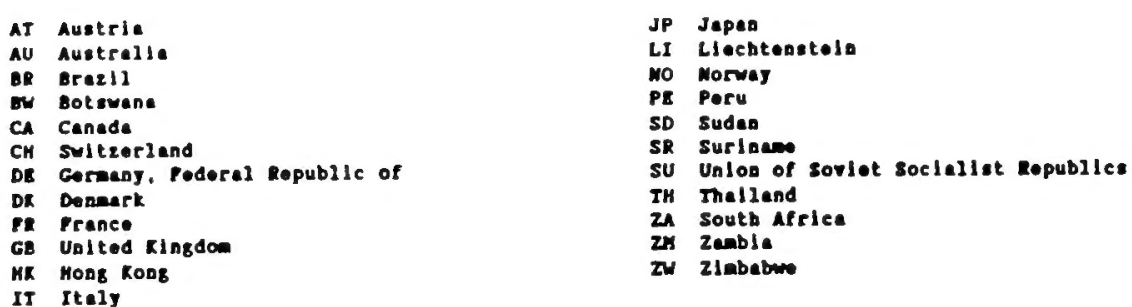



Prepared with financial support from the World Wildife Fund, the Irust Fund for the United Mations Bnvironment Stamp Conservation Fund and the United Nations Environment Programme.

A contribution to GEMS - the Global Environment Monitoring System.

The IUCN Conservation Monitoring Centre is grateful to the CITBS Secretariat for its assistance in the publication of this document.
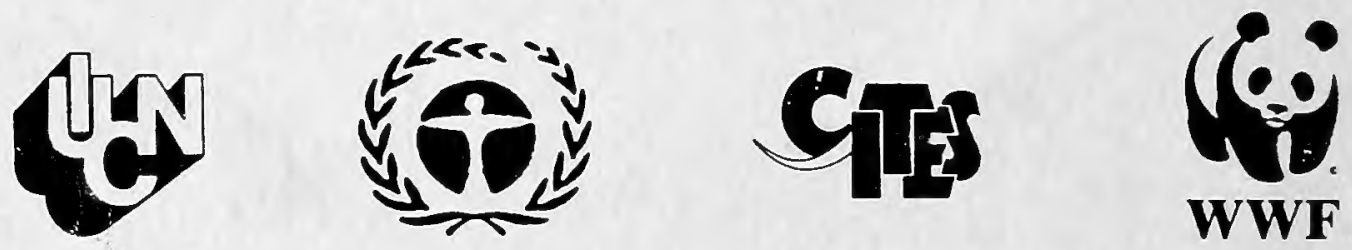